\title{
Shock sensitivity in the localised buckling of a beam on a nonlinear foundation: the case of a trenched subsea pipeline
}

\author{
Zhenkui Wanga,b , G.H.M. van der Heijden, ${ }^{\mathrm{b},}$, \\ ${ }^{a}$ State Key Laboratory of Hydraulic Engineering Simulation and Safety, Tianjin University, Tianjin 300072, China \\ ${ }^{\mathrm{b}}$ Department of Civil, Environmental and Geomatic Engineering, University College London, London WC1E 6BT, UK
}

\section{Corresponding author: G.H.M. van der Heijden, g.heijden@ucl.ac.uk}

\begin{abstract}
We study jump instability phenomena due to external disturbances to an axially loaded beam resting on a nonlinear foundation that provides both lateral and axial resistance. The lateral resistance is of destiffening-restiffening type known to lead to complex localisation phenomena governed by a Maxwell critical load that marks a phase transition to a periodic buckling pattern. For the benefit of having a concrete and realistic example we consider the case of a partially embedded trenched subsea pipeline under thermal loading but our results hold qualitatively for a wide class of problems with nonmonotonic lateral resistance. In the absence of axial resistance the pipeline is effectively under a dead compressive load and experiences shock-sensitivity for loads immediately past the Maxwell load, i.e., extreme sensitivity to perturbations as may for instance be caused by irregular fluid flow inside the pipe or landslides. Nonzero axial resistance leads to a coupling of axial and lateral deformation under thermal loading. We define a 'Maxwell temperature' beyond which the straight pipeline may snap into a localised buckling mode. Under increasing axial resistance this Maxwell temperature is pushed to higher (safer) values. Shock sensitivity gradually diminishes and becomes less chaotic: jumps become more predictable. We compute minimum energy barriers for escape from pre-buckled to post-buckled states, which, depending on the magnitude of the axial resistance, may be induced by either symmetric, or anti-symmetric or non-symmetric perturbations.
\end{abstract}

Keywords: Subsea pipelines; Thermal buckling; Localised lateral buckling; Nonlinear pipe-soil interaction model; Trench; Bifurcation; Maxwell load; Homoclinic snaking; Periodic buckling; Shock sensitivity.

\section{Introduction}

Beams and plates resting on a nonlinear foundation are known to buckle in a localised manner under (uni-)axial loading (Hunt et al., 1989; Kerr, 1978; Pocivavsek et al., 2008). If the foundation force is non-monotonic (of destiffening-restiffening characteristic) then typically at some point under increase of the load the localised buckle stops growing in amplitude and instead starts to spread, thereby creating a periodic pattern that gradually takes up most of the length of the structure (Hunt et al., 2000; Peletier, 2001). The phenomenon is governed by a Maxwell load that marks a 'phase transition' from the pre-buckled straight state to the periodic state. Similar behaviour of localisation followed by spreading is found in axially loaded cylinders buckling into a diamond-like pattern (Groh and Pirrera, 2019; Hunt et al., 1999), coiled twisted rods constrained to deform on a cylinder (van der Heijden et al., 2002), wrinkling of a thin film on a substrate (Jin et al., 2015) and folding of geological layers (Hobbs and Ord, 2012).

In most analytical work on these localisation phenomena only lateral resistance (elastic or frictional) is taken into account and the structure is free to slide in the axial direction. This frictionless sliding may be an acceptable approximation for the initial buckling pattern within a small-deflection theory as the amount of axial contraction goes as the deflection squared and is therefore small. However, as the buckling pattern spreads the end shortening can become large even for small deflections by the cumulative effect. Here we consider the case with axial resistance included using the subsea pipeline as a concrete and meaningful example.

Trenched subsea pipelines indeed offer an ideal example to explore the above complicated localisation phenomena in a realistic setting. In long pipelines localised buckling is the natural mode of buckling under the alignment conditions imposed by the longer structure at the ends of the buckle, and with the pipeline free to find its own lateral mobilisation length. In studies of pipeline buckling the effect of axial resistance has traditionally been included (Hobbs, 1984). Thermal effects as a 
result of axial friction lead to a coupling between axial and lateral deformation and we will explore its consequences for localised buckling.

Lateral foundation forces considered in the literature are often somewhat artificial, especially if they are treated as purely elastic. The pipeline problem offers a natural case for a realistic non-monotonic lateral resistance. Embedment of the pipeline, due to its own weight, produces a softening behaviour after breakout, while the trench walls give rise to restiffening behaviour at larger deflections. On the downside, due to the frictional nature of the resistance the proposed foundation characteristic is only valid in situations in which the displacements grow monotonically. We show that, nevertheless, valuable results can be obtained, by focussing on stability of the trivial state under finite perturbations (shocks).

The increasing global demand for oil and gas pushes the exploitation of hydrocarbon sources into ever deeper water. Long subsea pipelines are consequently becoming increasingly important for the transport of the hydrocarbon products from deep sea to the shore. To prevent solidification of the wax fraction in these products, subsea pipelines are required to operate under high-temperature and high-pressure conditions. This may lead to excessive axial compressive forces and localised lateral buckling is well-known to occur in exposed subsea pipelines (DNV-RP-F110, 2018). During their whole operational life pipelines undergo regular start-up and shut-down cycles. The resulting thermal cycles induce repeated localised buckling, which causes soil berms to accumulate in front of the pipeline's motion. This leads to increased soil resistance (Wang et al., 2017) and after several thermal cycles the pipeline appears to buckle in a trench. Another case is that the pipeline is laid in an open trench without backfill for mitigating hydrodynamic loads (DNV-RP-F109, 2011). Here we investigate the effect of the trench wall on lateral thermal pipeline buckling.

Much of the analytical research on lateral, as well as upheaval, subsea pipeline buckling in the literature is based on Hobbs's work (Hobbs, 1984). In this work the pipeline is modelled as a beam-column and the lateral resistance force is assumed to be constant, independent of the deflection. Based on this approach, Taylor and co-workers derive analytical solutions for ideal submarine pipelines by considering a deformation-dependent (nonlinear) resistance model (Taylor and Gan, 1986a) as well as analytical solutions for lateral and upheaval buckling of pipelines with initial imperfections (Taylor and Gan, 1986b). In (Hong et al., 2015) lateral buckling modes of pipelines with imperfection are compared against finite-element solutions. A nonlinear soil resistance model, allowing for partial pipeline embedment, is used in a lateral buckling analysis in (Zhang and Guedes Soares, 2019).

In all the above pipeline research the buckling mode is taken to be given by a solution of the linearised equations. The buckle profiles consist therefore of a (small) number of essentially sinusoidal lobes. It is good to realise though that localised buckling, with exponentially decaying deflection, is an intrinsic property of perfect elastic structures resting on a nonlinear foundation. This localised buckling is quite different from (Euler) column buckling. It is described by a Hamiltonian-Hopf bifurcation rather than the pitchfork bifurcation of column buckling. An important consequence is that unlike the critical load for column buckling, which depends strongly (quadratically) on the length of the structure, the critical load for localised buckling does not depend on this length (although the structure of course has to be long enough to support a localised buckle). The critical load for localised buckling is in fact lower than that for Euler buckling, although even this load is generally not reached as localised deflection is initiated by imperfections or perturbations.

Genuine localised buckling is considered in (Wang and van der Heijden, 2017) where we compute bifurcation diagrams of localised solutions (homoclinic orbits) for a partially embedded pipeline on an even seabed. Localised solutions are also considered in (Zhu et al., 2015) although the quoted boundary conditions do not maintain localisation as parameters of the system are varied. In (Zeng and Duan, 2014) a nonlinear and non-monotonic lateral soil resistance is employed to model partial embedment and homoclinic orbits are explicitly computed. Although the authors are not motivated by trenched pipelines, and do not consider thermal buckling, they find some of the homoclinic phenomena that we report on in this paper. However, they give limited physical interpretation of their results and do not investigate the stability implications.

Here we study localisation phenomena for a trenched pipeline with both lateral and axial resistance included. In the absence of axial resistance the pipeline is effectively under a dead compressive load. We identify a critical Maxwell point marking a 'phase transition' to a periodic buckling pattern with the pipeline bouncing between the trench walls. Associated with this critical point we find load-displacement curves with high sensitivity of solutions to small perturbations, giving rise to what 
has been called shock sensitivity (Thompson and van der Heijden, 2014). So, unlike the usual practice of reading loaddisplacement bifurcation diagrams, with or without imperfections included, as quasi-static processes that might encounter linear instability under infinitesimal perturbations, we are here interested in nonlinear instability phenomena with the pipeline being forced, by external finite disturbances, out of a linearly stable state and into another stable state. In particular, we are interested in the energy barrier, represented by an intermediate unstable 'mountain pass' state, to be overcome for such a transition from the straight pre-buckled state to a localised state, and its dependence on the axial resistance.

The organisation of the paper is as follows. In Section 2 we give details of the thermal, elastic and soil modelling of our trenched pipeline and identify the central Hamiltonian-Hopf bifurcation with associated homoclinic orbits describing localised buckling modes. In Section 3 we compute these homoclinic orbits and their bifurcation diagrams (load-displacement curves). By considering energy, in Section 4 we then discuss pipeline stability implications of these diagrams under various types of loading, dead, rigid and thermal. Thermal loading is found to interpolate between dead and rigid loading as the axial resistance increases from zero. In the process, shock sensitivity gradually diminishes. We quantify the shock sensitivity by computing energy barriers as a function of the axial soil resistance. Section 5 briefly discusses the dependence of our results on the breakout coefficient, which controls the non-monotonicity of the lateral resistance, and Section 6 closes the study with a summary and discussion.

\section{Problem modelling}

\subsection{Thermal pipeline buckling}

We imagine a pipeline laid in a trench and subjected to a total temperature difference $T_{0}$ between the fluid flowing inside the pipe and the environment. If the ends of the pipe are unrestrained then under an increase of the temperature difference the pipe will expand axially. This expansion will be resisted by friction between pipe and seabed (and surrounding soil). If the soil resistance for axial movement is constant, say $f_{A}$, then a compressive force will build up in the pipe, which will increase linearly with the distance from the freely-expanding end. At some point this compressive force is sufficient to halt further expansion of the central segment of the pipe. Thus an immobilised segment spreads from the centre of the pipe. The end points of this segment are called virtual anchor points. Between these points the compressive force in the pipe is equal to the force in a pipe with fixed ends under the same thermal load. Within the range of linear elastic response this compressive force can be written as

$$
P_{0}=E A \alpha T_{0}
$$

where $E$ is the elastic modulus, $A$ is the cross-sectional area of the pipeline and $\alpha$ is the coefficient of linear thermal expansion. Immobilisation will only occur if this compressive force is attained, which in the present scenario will only be the case if the length of the pipe is larger than $2 l_{i}$, where

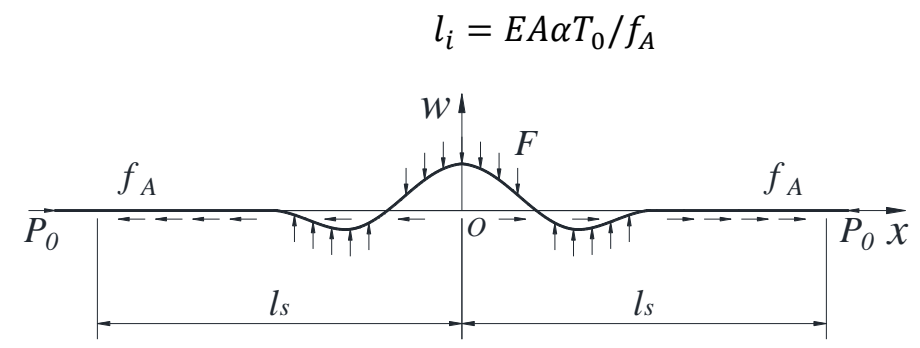

Fig. 1 Configuration and load distribution of localised lateral buckling.

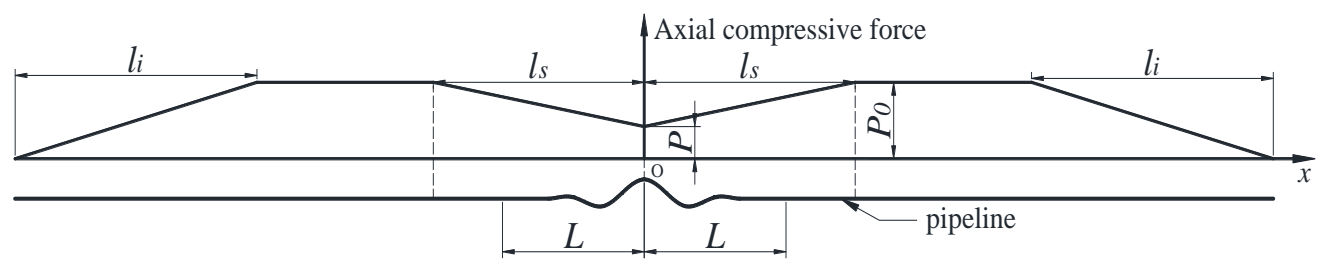

Fig. 2 Axial compressive force distribution of localised lateral buckling. 
Under increasing temperature difference, the compressive force $P_{0}$ increases and at some point buckling may be initiated. For a sufficiently long pipe this will be localised buckling, with exponentially decaying deflection. For a pipe without imperfections we expect this buckling to occur in the centre of the pipe, in a region of length $2 L$ (see Fig. 2). Here we shall assume this buckling to be lateral, i.e., horizontal, against the resistance of the surrounding soil, rather than vertical, against gravity. For normal coefficients of friction, the lateral mode occurs at a lower axial load than the vertical mode (Hobbs, 1984).

In the buckling process a central segment of the pipe will mobilise. The same scenario as described above then applies, but now in reverse. Thus, as pipe feeds into the buckle the compressive force in the pipe drops, pulling more pipe into the buckle. This feed-in will be halted at two more virtual anchor points, at compressive force $P_{0}$, bounding the mobilised region. Fig. 2 shows the feed-in region, of length $2 l_{s}(>2 L)$, within the larger immobilised pipe segment together with the localised buckle and the typical compressive force variation. In practice multiple (independent) localised buckles may form in the immobilised pipe segment, especially if it is long. In the following we present a theory for a single localised buckle.

\subsection{Governing pipeline equations}

We model the pipeline as an Euler-Bernoulli beam subjected to an axial compressive force. The lateral buckling shape of the pipeline is shown in Fig. 1. Note that by symmetry we need only consider half the length of the mobilised pipe $(0 \leq x \leq$ $l_{s}$ ). For the lateral and axial displacements, $w$ and $u$, we have the coupled equations (Kerr, 1978)

$$
\left\{\begin{array}{c}
\frac{\mathrm{d}^{2}}{\mathrm{~d} x^{2}}\left(E I \frac{\mathrm{d}^{2} w}{\mathrm{~d} x^{2}}\right)-\frac{\mathrm{d}}{\mathrm{d} x}\left[E A\left(\frac{\mathrm{d} u}{\mathrm{~d} x}+\frac{1}{2}\left(\frac{\mathrm{d} w}{\mathrm{~d} x}\right)^{2}-\alpha T_{0}\right) \frac{\mathrm{d} w}{\mathrm{~d} x}\right]+F(w)=0 \\
\frac{\mathrm{d}}{\mathrm{d} x}\left[E A\left(\frac{\mathrm{d} u}{\mathrm{~d} x}+\frac{1}{2}\left(\frac{\mathrm{d} w}{\mathrm{~d} x}\right)^{2}-\alpha T_{0}\right)\right]+f_{A}=0
\end{array}\right.
$$

up to leading nonlinear terms due to the nonlinear axial elastic strain $\varepsilon=\frac{\mathrm{d} u}{\mathrm{~d} x}+\frac{1}{2}\left(\frac{\mathrm{d} w}{\mathrm{~d} x}\right)^{2}$ as a result of lateral deflection. Here $E I$ is the bending stiffness with $I$ the second moment of area of the pipe's cross-section and $F$ is the nonlinear lateral soil resistance provided by the seabed and detailed in the next section. For the axial soil resistance (a force per unit length) we can write

$$
f_{A}=\mu_{A} W_{\text {pipe }}
$$

where $\mu_{A}$ is the axial friction coefficient between pipeline and seabed and $W_{\text {pipe }}$ is the submerged weight per unit length of the pipeline. We will take both elastic stiffnesses $E I$ and $E A$ to be constant.

In terms of the axial compressive force $\bar{P}=-E A\left(\varepsilon-\alpha T_{0}\right)$ we can solve the second equation in Eq. (3) as

$$
\bar{P}(x)=P+f_{A} x
$$

where $P$ is the compression at the centre of the pipe. Since at the anchor points the compression $\bar{P}(x)$ is $P_{0}$, we have

$$
P_{0}-P=f_{A} l_{S}
$$

The linear profile of the compressive force $\bar{P}(x)$ is sketched in Fig. 2.

By using Eq. (5) in the first equation of Eq. (3) we obtain a decoupled linear equation for the lateral deflection $w$. We simplify this equation further by making the assumption that the axial compressive force is constant in the buckling region $0 \leq x \leq L$ and equal to the force at the centre of the pipe, i.e., $\bar{P}=P$. This amounts to neglecting the axial resistance $f_{A}$ in the lateral buckle. This is standard practice in the pipeline and rail track literature (Hobbs, 1984; Hong et al., 2015; Kerr, 1978; Taylor and Gan, 1986a). An error analysis in (Wang et al., 2018), where solutions for minimum $(P)$ and maximum $\left(P_{0}\right)$ uniform compression in the buckled region are compared, shows that this assumption is acceptable. Further a posteriori justification is given in Section 4.3. For the lateral deflection we then have the constant-coefficient equation

$$
E I \frac{\mathrm{d}^{4} w}{\mathrm{~d} x^{4}}+P \frac{\mathrm{d}^{2} w}{\mathrm{~d} x^{2}}+F(w)=0 \quad(0 \leq x \leq L)
$$

Boundary conditions for Eq. (7), which must support localised solutions as shown in Fig. 1, will be discussed in detail in Section 2.4.

However, we make the above assumption only to compute $w$. For the axial deformation we solve the full equation. Thus, in the buckling region we solve 


$$
E A\left(\frac{\mathrm{d} u}{\mathrm{~d} x}+\frac{1}{2}\left(\frac{\mathrm{d} w}{\mathrm{~d} x}\right)^{2}-\alpha T_{0}\right)=-f_{A} x-P \quad(0 \leq x \leq L)
$$

with boundary condition $u(0)=0$, giving

$$
u(x)=-\frac{f_{A}}{2 E A}\left(x-l_{\mathrm{s}}\right)^{2}+\frac{f_{A} l_{S}^{2}}{2 E A}-\frac{1}{2} \int_{0}^{x}\left(\frac{\mathrm{d} w}{\mathrm{~d} x}\right)^{2} \mathrm{~d} x \quad(0 \leq x \leq L)
$$

In the slip region $\left(L \leq x \leq l_{\mathrm{s}}\right)$ we solve the same equation for $u$ but since now $w=0$, this becomes

$$
E A\left(\frac{\mathrm{d} u}{\mathrm{~d} x}-\alpha T_{0}\right)=-f_{A} x-P \quad\left(L \leq x \leq l_{\mathrm{s}}\right)
$$

Note that this satisfies the slip-length boundary condition $\frac{\mathrm{d} u}{\mathrm{~d} x}\left(l_{\mathrm{s}}\right)=0$ (Taylor and Gan, 1986b). A final integration subject to the boundary condition $u\left(l_{\mathrm{s}}\right)=0$ gives

$$
u(x)=-\frac{f_{A}}{2 E A}\left(x-l_{\mathrm{s}}\right)^{2} \quad\left(L \leq x \leq l_{\mathrm{s}}\right)
$$

Continuity of axial deformation at the end of the buckling region, $x=L$ (or $x=\tilde{L}$ for any $\tilde{L}>L$ ), requires

$$
\frac{f_{A} l_{S}^{2}}{2 E A}=\frac{1}{2} \int_{0}^{L}\left(\frac{\mathrm{d} w}{\mathrm{~d} x}\right)^{2} \mathrm{~d} x
$$

This condition can be interpreted as a deformational compatibility condition $u_{1}=u_{2}$ (Wang and van der Heijden, 2017). The left-hand side of Eq. (12) is the axial expansion

$$
u_{1}=\int_{0}^{l_{s}} \frac{\Delta \bar{P}(x)}{E A} \mathrm{~d} x
$$

with $\Delta \bar{P}(x)$ the drop in axial compressive force along the pipeline after buckling, given by

$$
\Delta \bar{P}(x)=f_{A}\left(l_{s}-x\right)
$$

The right-hand side of Eq. (12) is the geometric shortening $u_{2}$ as a result of the lateral deflection. Eq. (12) simply states that, since there are virtual anchor points at distance $l_{\mathrm{s}}$ from the centre of the pipe, the extra length of pipe in the buckle must come from axial expansion of the mobilised section of pipe.

From Eq. (12) we obtain an expression for $l_{s}$ :

$$
l_{s}=\sqrt{\frac{2 E A u_{2}}{f_{A}}}
$$

Combining this with Eq. (1) and Eq. (6), we finally obtain a relationship between $P$ and $T_{0}$ :

$$
T_{0}=\frac{\left(P+\sqrt{2 E A u_{2} f_{A}}\right)}{E A \alpha}
$$

For consistency we require $l_{s}$ to be larger than $L$ (see Fig. 2). Since there is no a priori guarantee that $l_{s}$ as computed from Eq. (15) satisfies this condition, we need to check any computed solutions for acceptability.

\subsection{Nonlinear lateral pipe-soil interaction}

For the pipe-soil interaction we construct a nonlinear model by combining a realistic model for the lateral resistance of a partially embedded pipeline on an even seabed with a stiffening characteristic for the trench walls. For the former we choose the model proposed by Chatterjee et al. (Chatterjee et al., 2012) on the basis of a detailed large-deformation finite-element analysis. This model simulates breakout resistance induced by the initial embedment as a result of partial penetration of the pipeline into the soil owing to its self-weight. Once breakout has occurred the lateral resistance drops and approaches a steady residual value. The model can be expressed by

$$
\mu_{1}=\frac{w}{|w|}\left(\mu_{\text {brk }}\left(1-\mathrm{e}^{-a_{1}\left(\frac{|w|}{D}\right)^{a_{2}}}\right)+\left(\mu_{\text {res }}-\mu_{\text {brk }}\right)\left(1-\mathrm{e}^{-a_{3}\left(\frac{|w|}{D}\right)^{a_{4}}}\right)\right)
$$

Here $\mu_{1}$ is the equivalent friction coefficient, $\mu_{b r k}$ is the breakout equivalent friction coefficient, $\mu_{r e s}$ is the residual equivalent friction coefficient and $D$ is the external diameter of the pipeline. The quantities $F_{1}=\mu_{1} W_{\text {pipe }}, F_{\text {brk }}=$ 
$\mu_{\mathrm{brk}} W_{\text {pipe }}$ and $F_{\text {res }}=\mu_{\text {res }} W_{\text {pipe }}$ are therefore, respectively, the lateral soil resistance, the breakout resistance and the residual resistance for a pipeline laid on an even seabed. $F_{1}$ captures both a frictional component of resistance below the pipe and a passive component required to lift and deform the region of soil in front of the pipe (White and Cheuk, 2008). The value of the coefficient $a_{3}$, which determines the distance required to mobilise the steady resistance, is in (Chatterjee et al., 2012) given in terms of the weight of the pipe $W_{\text {pipe }}$ and the vertical bearing capacity $V_{\max }$ as

$$
a_{3}=a_{5}\left(\frac{W_{\text {pipe }}}{V_{\max }}\right)+a_{6}
$$

where the values of the two further coefficients $a_{5}$ and $a_{6}$, for different values of the initial embedment $w_{\text {init }}$, are given by

$$
a_{5}=8.2 \frac{w_{\text {init }}}{D}-4.9, \quad a_{6}=-5.8 \frac{w_{\text {init }}}{D}+4.5
$$

We follow (Chatterjee et al., 2012) in choosing $a_{1}=25$ and $a_{4}=1.5$, but take $a_{2}=1$ in order to have a finite linear resistance $\left(k_{l}\right.$ in Section 2.4), which is physically realistic. We furthermore take $V_{\text {max }}=5 W_{\text {pipe }}$ and $w_{\text {init }}=0.3 \mathrm{D}$, so that $a_{3}=2.272$, and set $\mu_{\text {res }}=0.5$ and $\mu_{b r k}=2.1$. Of all these parameters only $\mu_{b r k}$ is varied in this paper (in Section 5).

The effect of the trench is not yet included in Eq. (17). We introduce a cubic nonlinearity to simulate the steeply rising soil resistance provided by the wall of the trench when the buckled pipe contacts the wall. The corresponding friction coefficient of this cubic nonlinearity term is expressed by

$$
\mu_{2}=k \frac{w}{|w|}\left(\frac{|w|-d}{D}\right)^{3} \quad(|w| \geq d)
$$

where $k$ is a 'softness' parameter that controls the steepness of the trench wall resistance, while $d$ represents the width of the trench. This additional trench force only acts if the displacement $|w|$ exceeds $d$.

Combining Eq. (17) and Eq. (20), the friction coefficient of the whole nonlinear lateral pipe-soil interaction model for the pipeline, as shown in Fig. 3, is

$$
\mu=\left\{\begin{array}{cc}
\mu_{1} & |w|<d \\
\mu_{1}+\mu_{2} & |w| \geq d
\end{array}\right.
$$

The nonlinear lateral soil resistance for the pipeline can then be calculated by

$$
F=\mu W_{\text {pipe }}
$$

Note that $F$ is continuous and (twice) differentiable at $|w|=d$. The non-monotonic softening-restiffening characteristic of the soil resistance $F$, combining the effects of embedment and trenching, will play an important role in determining localised buckling patterns.

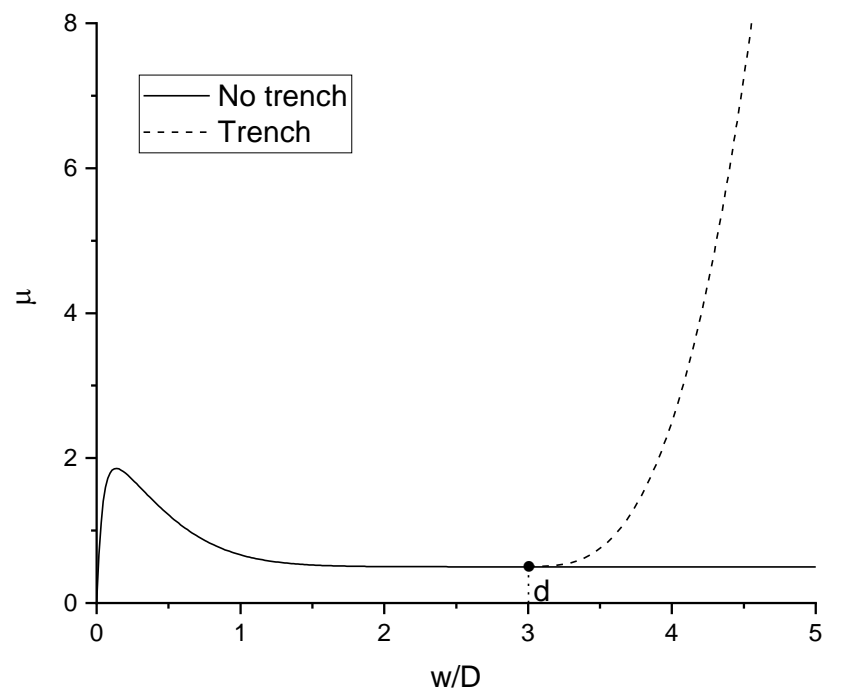

Fig. 3 Nonlinear lateral soil resistance model. $\left(d=3 \mathrm{D}, k=2, \mu_{b r k}=2.1.\right)$ 


\subsection{Localised buckling}

Here we discuss the localised solutions of Eq. (7) with $F$ as given in Eq. (22). It is useful to rewrite the fourth-order Eq. (7) as an equivalent four-dimensional system of first-order equations (writing $w=w_{1}$ )

$$
\left\{\begin{array}{c}
\frac{\mathrm{d} w_{1}}{\mathrm{~d} x}=w_{2} \\
\frac{\mathrm{d} w_{2}}{\mathrm{~d} x}=w_{3} \\
\frac{\mathrm{d} w_{3}}{\mathrm{~d} x}=w_{4} \\
\frac{\mathrm{d} w_{4}}{\mathrm{~d} x}=-\frac{1}{E I}\left(P w_{3}+F\left(w_{1}\right)\right)
\end{array}\right.
$$

This four-dimensional system is a Hamiltonian system (when viewed in appropriate coordinates) with Hamiltonian

$$
H=\frac{1}{2} E I w_{3}^{2}-E I w_{2} w_{4}-\frac{1}{2} P w_{2}^{2}-\int_{0}^{w_{1}} F(y) \mathrm{d} y
$$

This $H$ is therefore a conserved quantity, meaning that it is independent of $x$ along solutions of the system of equations. (That $H$ is constant can be verified directly by differentiating $H$ in Eq. (24) with respect to $x$ and substituting from Eq. (23) to show that $\mathrm{d} H / \mathrm{d} x=0$.)

Solutions of Eq. (23) are orbits in a four-dimensional phase space with coordinates $\left(w_{1}, w_{2}, w_{3}, w_{4}\right)$. The straight pipe solution is represented by the fixed point $j=(0,0,0,0)$. The Hamiltonian in this fixed point has value $H=0$. The eigenvalues of the fixed point are

$$
\pm \mathrm{i} \sqrt{\frac{P \pm \sqrt{P^{2}-4 E I k_{l}}}{2 E I}}
$$

where, since $a_{2}=1, k_{l}=\left(\frac{\mathrm{dF}}{\mathrm{dw}}\right)_{w=0}=\frac{a_{1} \mu_{\mathrm{brk}} W_{\text {pipe }}}{D}$. We conclude that at the critical load $P=P_{c r}$, where

$$
P_{c r}=2 \sqrt{k_{l} E I}
$$

the eigenvalues change from a quadruple of complex eigenvalues to two complex conjugate pairs of imaginary eigenvalues (see Fig. 4). This is called a Hamiltonian-Hopf bifurcation (Hunt et al., 1989; van der Heijden et al., 1998) and marks the loss of (linear) stability of the straight solution. For $P<P_{c r}$ the eigenvalues in Eq. (25) can be written as $\pm \lambda \pm i \omega$, with real $\lambda$ and $\omega$ given by

$$
\lambda=\frac{\sqrt{2 \sqrt{E I k_{l}}-P}}{2 \sqrt{E I}}, \quad \omega=\frac{\sqrt{2 \sqrt{E I k_{l}}+P}}{2 \sqrt{E I}}
$$

For comparison, the critical load for buckling of a pinned-pinned beam of given length $L$ into a pattern of $n$ half sine waves is

$$
P_{c r, p e r i o d i c}=\frac{n^{2} \pi^{2} E I}{L^{2}}+\frac{k_{L} L^{2}}{n^{2} \pi^{2}}
$$

It is straightforward to show that $P_{c r} \leq P_{c r, p e r i o d i c}$ for all $n$.

The symmetry and multiplicity of bifurcating solutions is governed by the symmetry of the system of equations. We have the following two reversing symmetries, i.e., the equations are invariant under the following simultaneous sign changes:

$$
\begin{aligned}
& R_{1}: x \rightarrow-x,\left(w_{1}, w_{2}, w_{3}, w_{4}\right) \rightarrow\left(w_{1},-w_{2}, w_{3},-w_{4}\right) \\
& R_{2}: x \rightarrow-x, \quad\left(w_{1}, w_{2}, w_{3}, w_{4}\right) \rightarrow\left(-w_{1}, w_{2},-w_{3}, w_{4}\right)
\end{aligned}
$$

It is well-known that among the solutions bifurcating from the trivial straight solution into the region of the complex quadruple of eigenvalues (here for $P<P_{c r}$ ) are so-called homoclinic orbits that leave the unstable fixed point in the plane spanned by the eigenvectors corresponding to the unstable eigenvalues (with positive real part), make a large excursion in the phase space and then return to the fixed point in the plane spanned by the eigenvectors corresponding to the stable eigenvalues (with negative real part) (Champneys and Spence, 1993; van der Heijden et al., 1998). These solutions thus approach the straight solution in both limits $x \rightarrow \pm \infty$ and are therefore also called localised solutions. We are here interested in these localised solutions and therefore apply what might be called homoclinic boundary conditions: we take the end points of the solution to be close to the fixed point $j$, thereby ensuring straight tails. Since we have no control over the length $L$, for this 
approach to give good solutions we need to assume the pipe to be long enough that solutions decay to zero towards the ends. This is reasonable in thermal pipeline buckling in which a relatively small segment of pipe, of unknown length, buckles within an effectively infinitely long immobilised pipeline providing alignment end conditions. The boundary conditions of thermal pipeline buckling are thus 'naturally homoclinic'.

Because of the above reversing symmetries, both a symmetric ( $R_{1}$-reversible) and an anti-symmetric $\left(R_{2}\right.$-reversible) solution bifurcate. Half these localised solutions are shown in Fig. 5(a), while the corresponding half orbits in (a twodimensional projection of) the phase space are shown in Fig. 5(b). Note that the homoclinic orbits spiral out of (and back into) the fixed point because of the complex eigenvalues. By continuity it follows therefore that along these localised solutions $H=0$, since the fixed point has this Hamiltonian value.

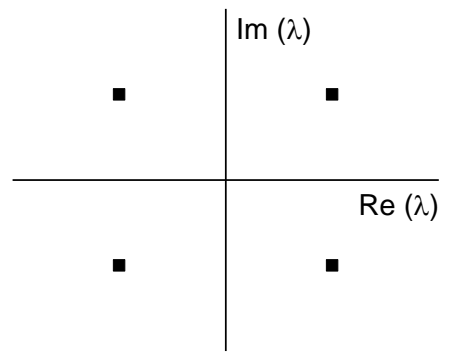

(a)

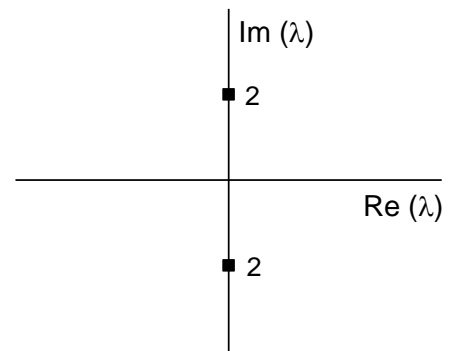

(b)

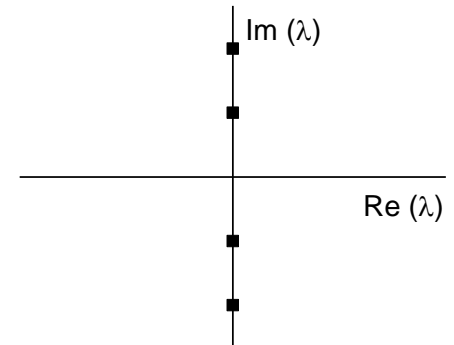

(c)

Fig. 4 The behaviour of eigenvalues at the Hamiltonian-Hopf bifurcation. (a) $P<P_{c r}$. (b) $P=P_{c r}$. (c) $P>P_{c r}$.

Table 1. Design parameters.

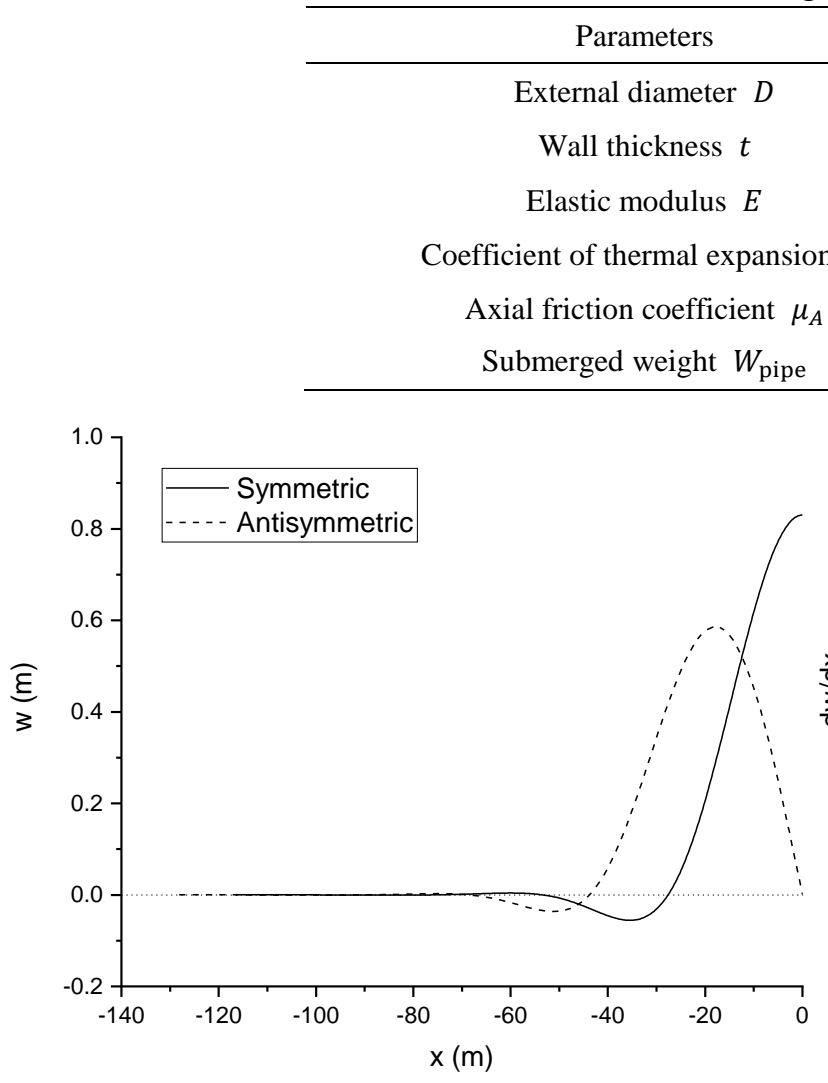

(a)

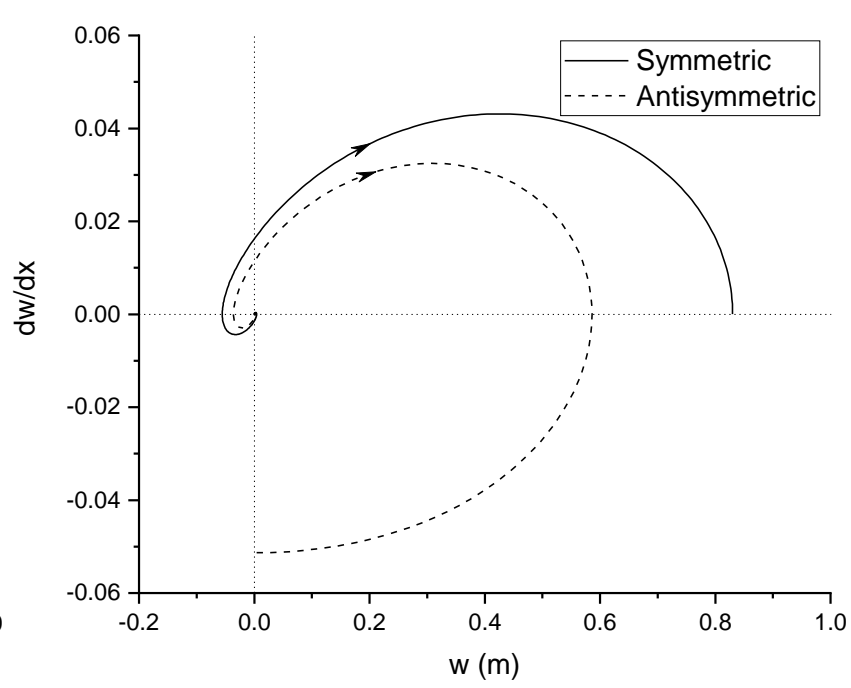

(b)

Fig. 5 Typical (half) solutions obtained by the shooting method. (a) Deformed shapes. (b) Homoclinic orbits in phase space. $\left(d=3 \mathrm{D}, k=2, \mu_{b r k}=2.1, P=4.2 \mathrm{MN}\right.$. $)$ 


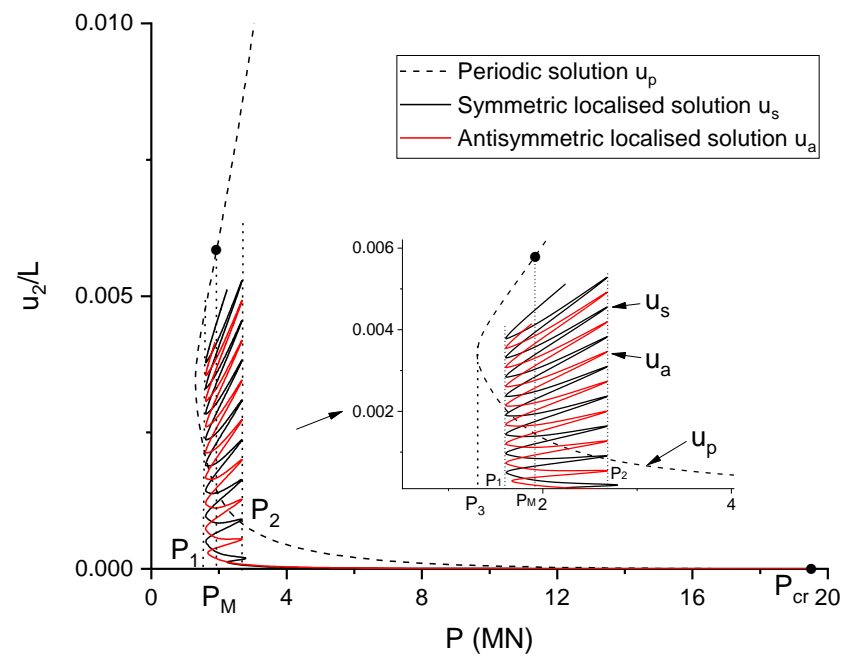

(a)

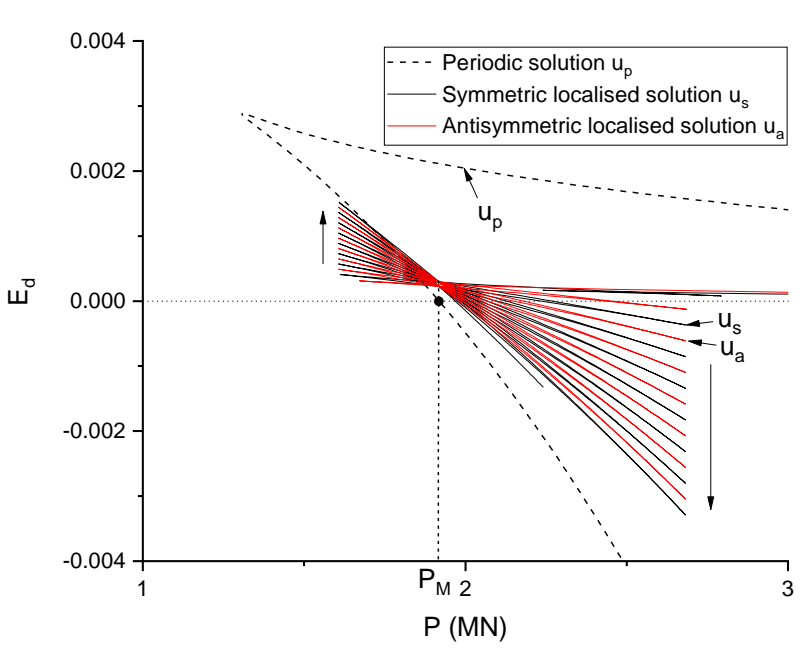

(b)

Fig. 6 (a) Bifurcation diagram with branches of homoclinic and periodic solutions, with Hamiltonian $H=0$, bifurcating subcritically at the critical (Hamiltonian-Hopf) load $P_{c r}=19.51 \mathrm{MN} . P_{M}=1.9181 \mathrm{MN}$ is the Maxwell load. (b) Total potential energy of the homoclinic and periodic solutions. $\left(d=3 \mathrm{D}, k=2, \mu_{b r k}=2.1\right.$. $)$

\section{Homoclinic solutions and their bifurcations}

For $P<P_{c r}$, we compute approximate (half) homoclinic solutions as shown in Fig. 5(a) by formulating a shooting problem on a truncated $x$ interval $[-L, 0]$. Here $L$, the half length of the homoclinic solution, is chosen large enough that the solution is well-localised in the sense that it is very nearly decayed to the trivial straight solution $j$ at $x=-L$. For details of the shooting method we refer to (Wang and van der Heijden, 2017). The (realistic) physical parameters used in this study are listed in Table 1. For these parameters and the additional choice $d=3 D, k=2$ and $\mu_{\text {brk }}=2.1$ of Fig. 3, we have $k_{l}=$ $3.06923 \times 10^{5} \mathrm{~N} / \mathrm{m}$. For the case $P=4.2 \mathrm{MN}$, shown in Fig. $5, L=116.59001 \mathrm{~m}$ for the symmetric and $L=$ $128.12790 \mathrm{~m}$ for the anti-symmetric homoclinic solution. From Eq. (15) we also obtain $l_{s}=308.68224 \mathrm{~m}$ for the symmetric solution and $l_{s}=356.79818 \mathrm{~m}$ for the anti-symmetric solution, noting that they are larger than $L$, as required (see Fig. 2).

Fig. 6 illustrates the bifurcation diagram, obtained by parameter continuation, showing the branches $u_{s}$ and $u_{a}$ of symmetric and anti-symmetric homoclinic orbits bifurcating from the critical load $P_{c r}$ (in fact, pairs of branches of $R_{1^{-}}$, respectively $R_{2}$-symmetric homoclinic orbits). The plot in Fig. 6 confirms that the post-buckling localised solutions exist for loads smaller than the critical load $P_{c r}$, which, from Eq. (26), is $P_{c r}=19.51 \mathrm{MN}$, i.e., the localised solutions bifurcate subcritically.

At lower values of $P$ the branches of localised solutions in Fig. 6 start to oscillate upwards in what is known as a homoclinic tower (Avitabile et al., 2010; Burke and Knobloch, 2006, 2007; van der Heijden et al., 2002; Woods and Champneys, 1999). As we go up the tower the localised solutions acquire more and more periods (oscillations), as illustrated in Fig. 7 and Fig. 8 for the symmetric solutions, eventually taking up almost the entire homoclinic orbit leaving only small straight tails at the ends. (Parameter continuation in $L$ has been used to present all localised solutions in Fig. 8 with the same length $L=1200 \mathrm{~m}$.) Each successive fold (saddle-node bifurcation) in the bifurcation diagram adds half a period at each end to the solution, leading to greater end shortening $u_{2}$. Left-opening folds (and right-opening folds) occur very nearly at identical $P$ values, but close inspection shows that folds higher up the tower occur for smaller $P$. The solution measure plotted in Fig. 6 is the normalised end shortening $u_{2} / L$, where $L=1200 \mathrm{~m}$, chosen to be slightly larger than the length of the periodic segment for the localised solutions at the top of the tower.

For finite length $L$ of the localised solution only a finite number of periods can be accommodated and therefore only a finite number of wiggles can be computed in the tower while maintaining the homoclinic boundary conditions. For an infinitely long pipeline infinitely many wiggles would appear and the values of $P$ where a fold occurs would asymptotically approach values $P_{1}$ and $P_{2}$ (monotonically and from the right), which we estimate in Fig. 6 to be $P_{1}=1.5823 \mathrm{MN}$ and 
$P_{2}=2.7086 \mathrm{MN}$. On the interval $\left[P_{1}, P_{2}\right]$ there would be infinitely many localised solutions that would approach a fullydeveloped periodic solution, with finite value of the normalised end shortening $u_{2} / L$ as $L \rightarrow \infty$.

Fig. 9 shows different localised solutions on the same branch of the tower between two successive folds. It is seen that under increasing compressive load $P$ the amplitude of the periodic segment increases while the period decreases, as one would expect. A comparison of the amplitudes of solutions in Fig. 8 and Fig. 9 with Fig. 3 reveals that the pipeline in these solutions is pressed firmly against the trench wall.

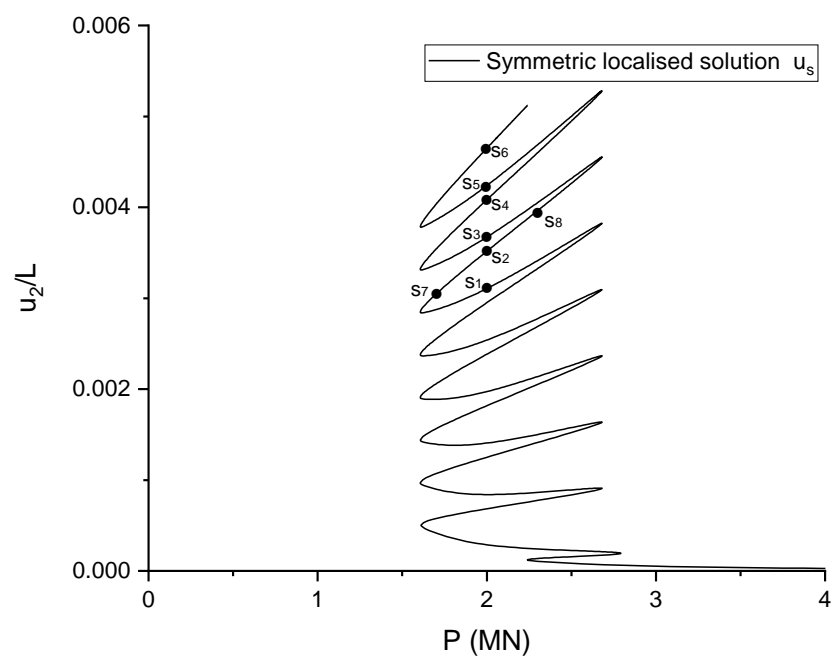

Fig. 7 Close-up of the bifurcation diagram for the symmetric localised solution in Fig. 6(a). Labels mark the locations of the localised solutions shown in Fig. 8 and Fig. 9.
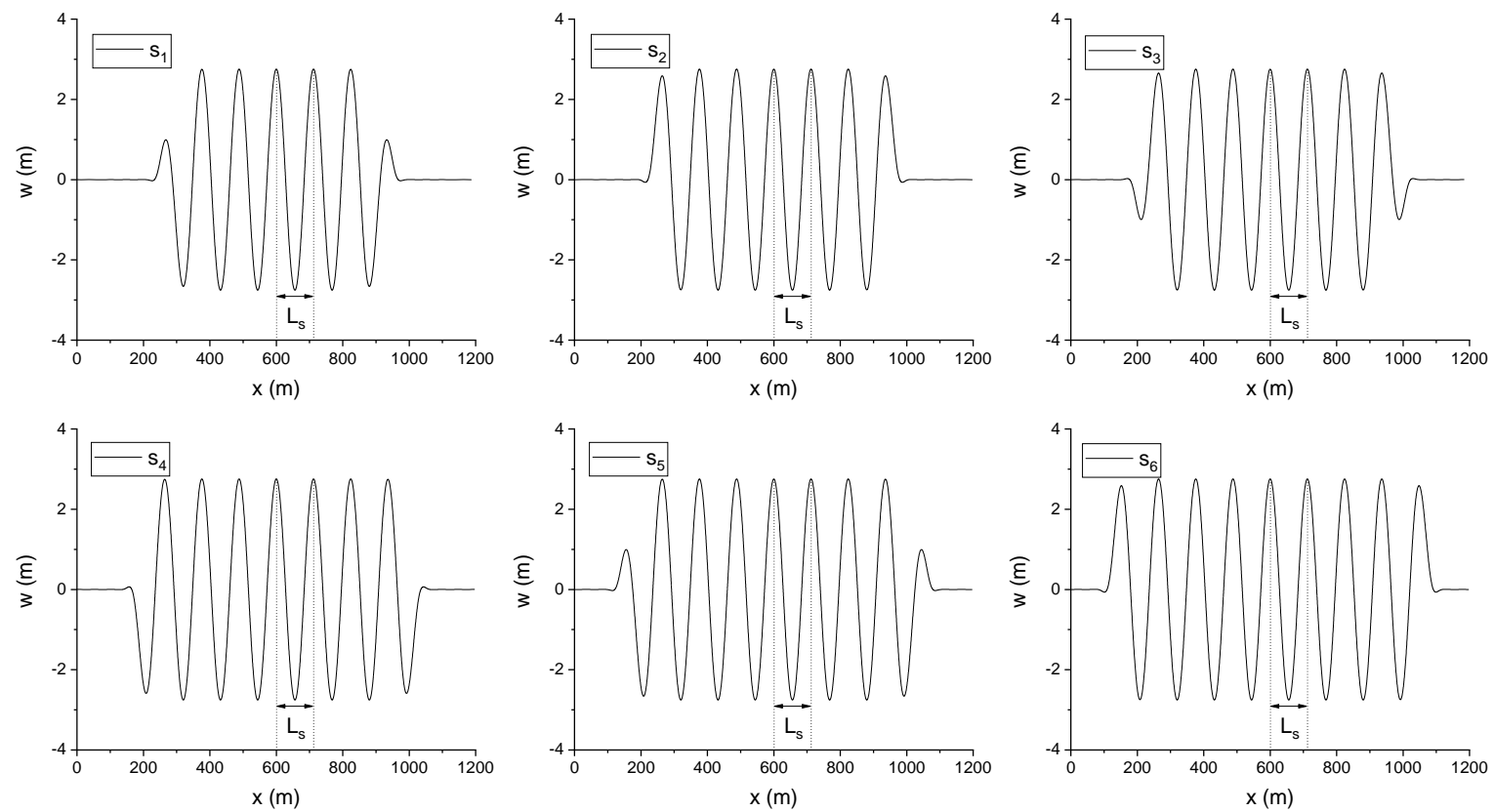

Fig. 8 Sample shapes of symmetric localised solutions indicated in Fig. 7. $\left(d=3 \mathrm{D}, k=2, \mu_{b r k}=2.1, P=2 \mathrm{MN}\right.$. 


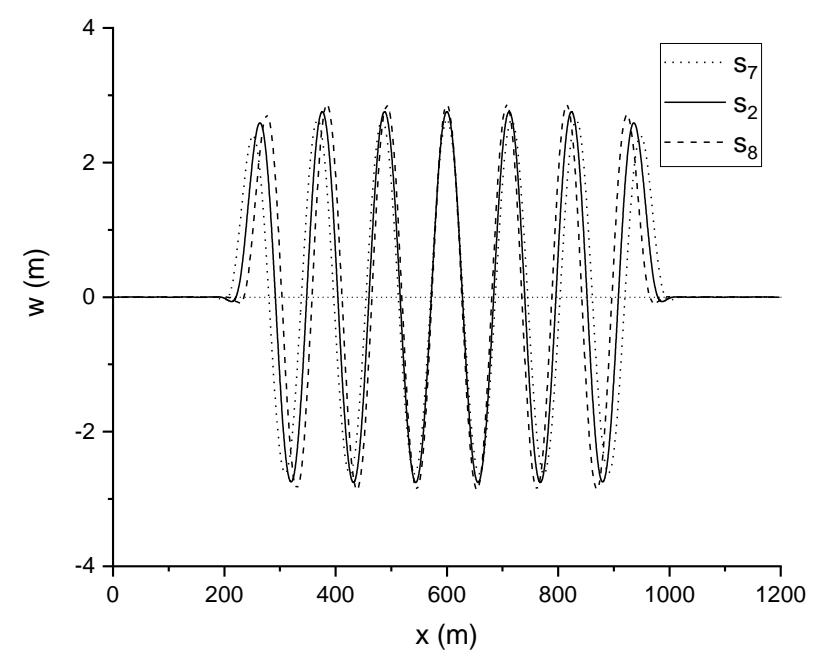

Fig. 9 Sample shapes of symmetric localised solutions along a single branch in Fig. 7. $\left(d=3 \mathrm{D}, k=2, \mu_{b r k}=2.1\right.$,

$$
P\left(s_{7}\right)=1.7 \mathrm{MN}, P\left(s_{2}\right)=2 \mathrm{MN}, P\left(s_{8}\right)=2.3 \mathrm{MN} \text {.) }
$$

Because the localised solutions have $H=0$, the embedded periodic solutions must also have $H=0$. Also shown in Fig. 6 is a branch $u_{p}$ of periodic solutions, having $H=0$, that branches from the Hamiltonian-Hopf bifurcation and intersects the tower of localised solutions. To draw this curve of periodic solutions in Fig. 6, $L$ is taken equal to their (varying) period. It is these periodic solutions at the top of the tower that are approached by the localised solutions. The black dot on the branch $u_{p}$ indicates the periodic solution with the same total potential energy density as the pre-buckled straight solution. This energy density (i.e., energy per unit length) can be written as

$$
E_{d}=E_{b}+E_{f}+E_{P}=\frac{1}{L} \int_{0}^{L}\left(\frac{1}{2} E I w_{3}^{2}+\int_{0}^{w_{1}} F(y) \mathrm{d} y-\frac{1}{2} P w_{2}^{2}\right) \mathrm{d} x
$$

where the first term, $E_{b}$, is the bending energy, the second term, $E_{f}$, is the foundation energy and the third term, $E_{P}$, is the work done by the compressive load $P$. Note that the energy density of the straight solution is zero.

The critical load where the periodic solution has the same energy density as the pre-buckled straight solution is called the Maxwell load $P_{M}$, as indicated in Fig. $6(\mathrm{~b})$; numerically, $P_{M}=1.9181 \mathrm{MN}$. It marks a 'phase transition' from the straight state to a periodic state in which the pipeline bounces back and forth between the trench walls.

Because the Hamiltonian-Hopf bifurcation is subcritical, both localised and periodic solution branches initially carry unstable solutions but stabilise at the first fold, and for the localised solutions then change stability at each successive fold. Stability of the various solutions (under infinitesimal perturbations) is therefore as indicated in Fig. 12, which we discuss in more detail later. Note that the fold along $u_{p}$, at $P=P_{3}$, implies that the periodic solution at the black dot in Fig. 6 is stable.

The interpretation of these bifurcation diagrams needs some care. The resistance $F$ as defined in Section 2.3 is positive for $w>0$ and negative for $w<0$ (in both cases resisting the deformation, as illustrated in Fig. 1). It therefore describes friction only if the pipeline 'tends to' increase its deflection (for all $x$ ). Now, one likes to interpret load-displacement bifurcation diagrams as quasi-static loading processes. This interpretation is only valid here if the deflection $|w|$ increases. It is good to realise, though, that each point along the bifurcation diagram individually, at certain load $P$, is a valid equilibrium solution under the corresponding force $F$. From this solution we can then increase the load $P$, causing the lateral deflection $|w|$ to increase. If we then encounter a jump to another solution (for instance, at a fold) in which $|w|$ decreases then this new solution need not lie on the bifurcation diagram, but if we subsequently increase $P$ again and the deflection starts to increase then the solution jumps back onto the computed bifurcation diagram (the friction force simply changes sign in the process).

In conclusion, we can interpret bifurcation diagrams as quasi-static processes only if we avoid reversals of the deformation. This is, however, not much of a restriction because in this work we are primarily interested in jumps of the pipeline out of its straight configuration and into a post-buckled localised state, as induced by external disturbances. For these individual localised states the lateral friction force is valid, as is the energy barrier computed for the jump. All stable solutions in our bifurcation diagrams are legitimate possible destinations for the pipeline when perturbed out of its stable straight 
configuration. Of course, if the frictional component of $F$ is negligible and $F$ may be interpreted as an elastic resistance then we are allowed to vary $P$ in both directions and any interpreted quasi-static loading sequence in our bifurcation diagrams is entirely physical.

In addition to symmetric and anti-symmetric localised solutions the equations also have non-symmetric localised solutions. These exist on branches connecting pairs of pitchfork bifurcations on the symmetric and anti-symmetric branches (see Fig. 10). These connecting branches (in fact, four branches of $R_{1}$-symmetric and $R_{2}$-symmetric pairs of solutions, all having the same end shortening $u_{2}$ ), sometimes called 'rungs' (Burke and Knobloch, 2007), may be computed by replacing the symmetry conditions at $x=0$ (see Fig. 5) by boundary conditions over the entire interval $[-L, L]$ so that non-symmetric solutions are allowed. All non-symmetric solutions are unstable (Burke and Knobloch, 2007). Solutions at labels 'a', 'b', 'c', 'd', 'e' and ' $\mathrm{f}$ ' in Fig. 10 are displayed in Fig. 11. It is seen that the non-symmetric solutions (' $b$ ' and 'e', at $P=2 \mathrm{MN}$ ) 'interpolate' between symmetric and anti-symmetric solutions by gradually deforming their shape.

We remark here also that in addition to these non-symmetric solutions there are multi-pulse localised solutions consisting of multiple copies of the single-pulse solutions computed here and lying on curves that also run up the homoclinic tower (Knobloch, 2015; Knobloch et al., 2011; van der Heijden et al., 2002). Since these solutions have correspondingly higher energy they will play no role in our stability discussion later and we will not further consider them.

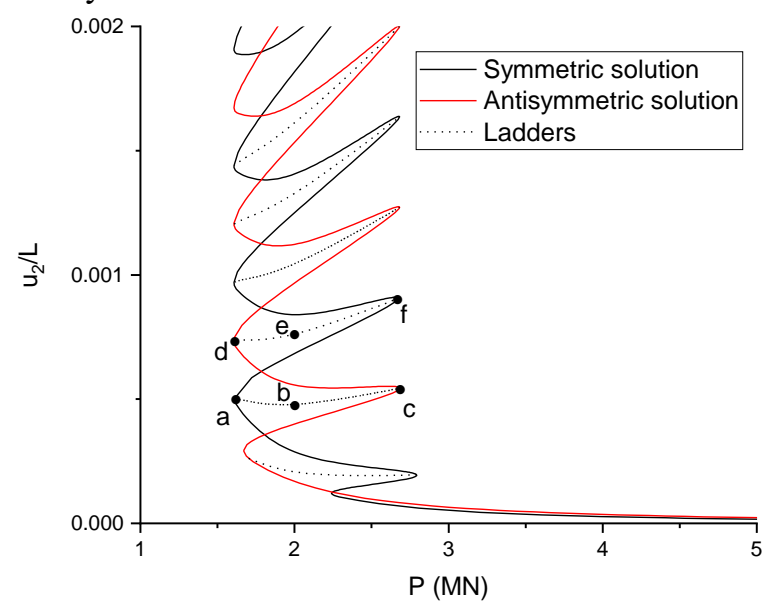

Fig. 10 Bifurcation diagram with 'rungs' connecting the symmetric and anti-symmetric localised solution branches. The labels ' $\mathrm{a}$ ' to ' $\mathrm{f}$ ' mark the locations of the solutions shown in Fig. 11. $\left(d=3 \mathrm{D}, k=2, \mu_{\text {brk }}=2.1\right.$.)
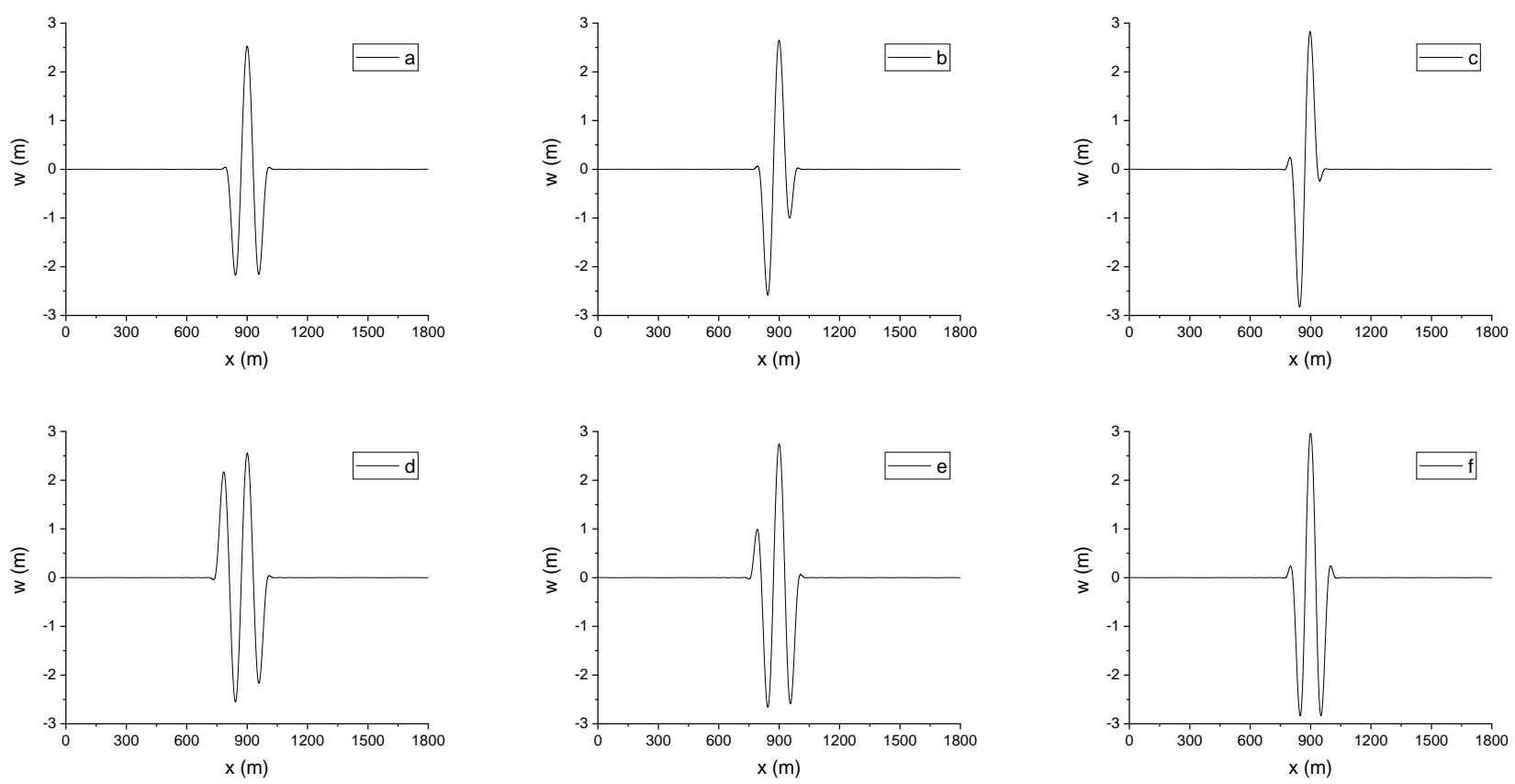

Fig. 11 Solution shapes at points indicated in Fig. 10 showing non-symmetric solutions intermediate between symmetric and anti-symmetric solutions. $\left(d=3 \mathrm{D}, k=2, \mu_{b r k}=2.1\right.$. $)$ 


\section{Stability under finite disturbances for various types of loading}

The complicated intertwining bifurcation behaviour in Fig. 6 and Fig. 10 is familiar from previous studies, especially those of the Swift-Hohenberg equation, and is commonly referred to as homoclinic snaking (Avitabile et al., 2010; Burke and Knobloch, 2006, 2007; van der Heijden et al., 2002; Woods and Champneys, 1999). An explanation of the behaviour in terms of the underlying phase-space dynamics as well as a physical interpretation is given in the Appendix. This in particular clarifies why a branch of periodic solutions like $u_{p}$ exists. Here we continue with an analysis of the stability implications for our pipeline problem. Although temperature $T_{0}$ is the control parameter in thermal pipeline buckling, if the axial resistance $f_{A}$ is negligible then, according to Eq. (16), controlling $T_{0}$ is equivalent to controlling the compression $P$ at the centre of the pipe. We will therefore first consider the case of dead compressive loading in which $P$ is the control parameter (Section 4.1). It will also be instructive to consider the case of rigid loading in which the end shortening $u_{2}$ is controlled. In view of Eq. (16) this corresponds to the other extreme of large $f_{A}$. We consider this case in Section 4.2. In Section 4.3 we finally deal with thermal loading by considering intermediate values of axial resistance, thereby interpolating between the extremes of dead and rigid loading.

\subsection{Dead loading ( $P$ control)}

To be able to give a stability discussion we compute the total potential energy $E_{d}$ given in Eq. (31). Fig. 6(b) shows $E_{d}$ against $P$ along the branches of localised and periodic solutions in Fig. 6(a). Recall that the straight pipeline has $E_{d}=0$. For small $P$ the straight solution will be stable. Indeed Fig. 6(b) shows that all other solutions have energy $E_{d}>0$. At the Maxwell load $P_{M}$ the periodic solution has the same energy as the straight solution (by definition). For $P>P_{M}$ the periodic solution has lower energy (while the localised solutions initially continue to have higher energy). The straight solution is still locally stable (and remains so for loads all the way up to $P_{c r}$ ), but is no longer globally stable. It is called metastable. Under the right perturbation the pipeline may be forced into the lower-energy periodic state. We will obtain an estimate for the minimum energy required to induce this transition.

Fig. 12 shows enlargements of the bifurcation diagram and energy plot in Fig. 6 for the symmetric localised solution and the periodic solution, with stability under dead loading indicated. In Fig. 12(b) we see that stable (solid) branches in the bifurcation diagram rotate (approximately) in clockwise direction about a point labelled $c_{1}$, while unstable (dashed) branches rotate (approximately) about point $c_{2}$, the latter having higher energy. As a result of this rotating motion successive stable branches in the homoclinic tower $\left(n_{1}-n_{2}, n_{3}-n_{4}, n_{5}-n_{6}, \ldots\right)$ intersect each other. This means that at the corresponding $\mathrm{P}$ values successive stable branches have solutions with the same energy $E_{d}$. At these $P$ values, therefore, transitions between stable localised solutions with different number of periods in the periodic segment are possible at relatively low energy cost.

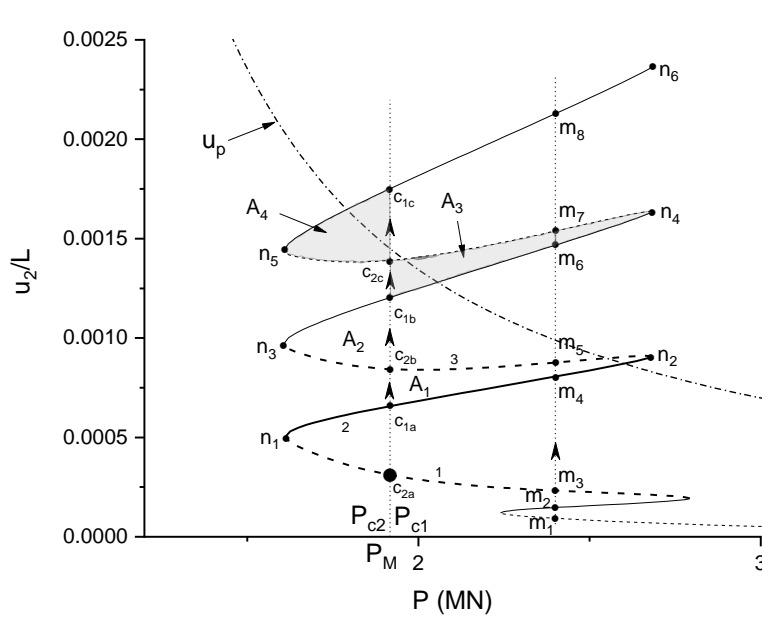

(a)

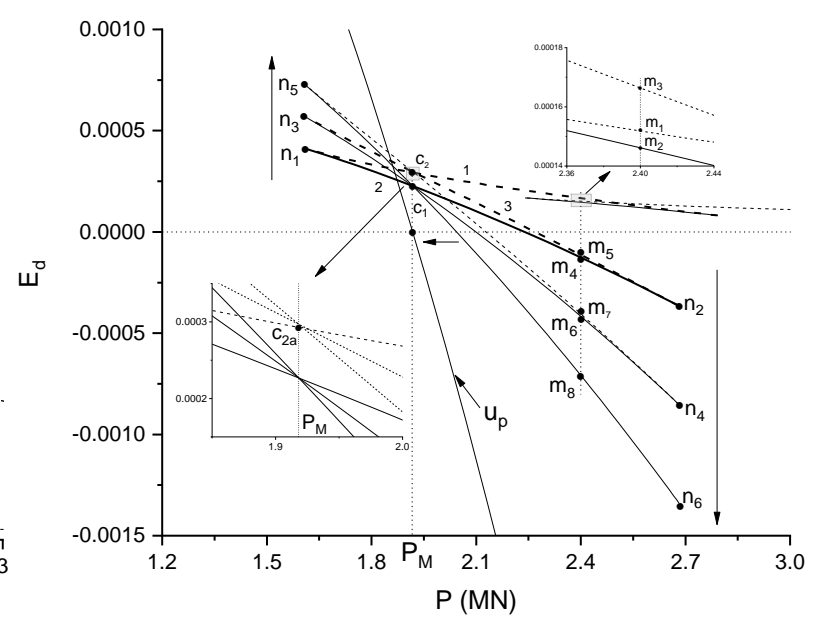

(b)

Fig. 12 Stability analysis of homoclinic orbits under dead loading conditions (solid lines indicate stable solutions, dashed lines unstable solutions). The big black dot indicates the 'mountain pass' state with lowest energy barrier for escape from the trivial straight solution. (a) Close-up of the bifurcation diagram of symmetric localised solutions in Fig. 6(a). (b) Closeup of the total potential energy plot in Fig. $6(\mathrm{~b}) .\left(d=3 \mathrm{D}, k=2, \mu_{b r k}=2.1.\right)$ 


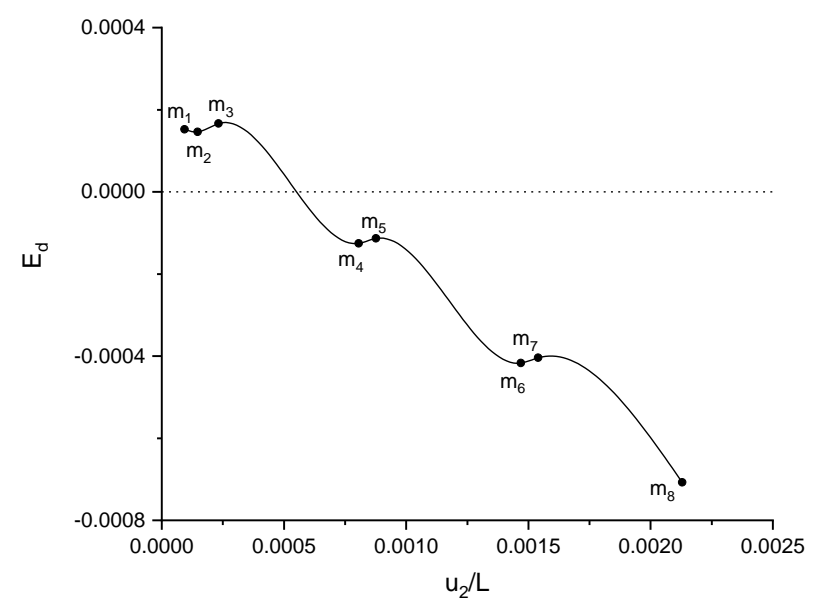

Fig. 13 Energy landscape at a constant axial compressive force of $P=2.4 \mathrm{MN}$. The labels in this figure correspond to the labels in Fig. 12.

Two such transitions $\left(c_{1 a}-c_{1 b}\right.$ at $P=P_{\mathrm{c} 1}=1.91829 \mathrm{MN}$ and $c_{1 b}-c_{1 c}$ at $\left.P=P_{\mathrm{c} 2}=1.91822 \mathrm{MN}\right)$ are indicated by arrows in Fig. 12(a). These vertical jumps occur at such points that equal areas $A_{\mathrm{i}}$ are cut off in this $P$ - $u_{2}$ diagram in which enclosed area represents potential energy. For instance, the coloured area $A_{3}$ represents the energy barrier to be overcome to escape from the stable solution labelled $c_{1 b}$ via the unstable saddle-type 'mountain pass' state $c_{2 c}$. The equal area $A_{4}$ then represents the energy released in 'descending' from the unstable state $c_{2 c}$ to the stable solution $c_{1 c}$ of the same energy as $c_{1 b}$, but with one more period. This equal-area rule is analogous to the Maxwell construction for isotherms in pressure-volume phase diagrams (or similar diagrams of conjugate variables) in thermodynamics (Reichl, 2016).

It is good to stress here that the direct vertical path from $c_{1 b}$ to $c_{1 c}$ is not an equilibrium path. In reality the transition will occur as a dynamic jump not covered by this statics theory. This will involve vibrations that need to be damped out if we are to end up on the equilibrium curve. So energy will be dissipated and we need to jump sooner to end up in the destination state. We ignore these dynamical effects here by pretending that the transition occurs quasi-statically.

The areas separated by $P_{\mathrm{c} 2}$ are by direct numerical calculation found to be $A_{3}=7.00 \times 10^{-5}$ and $A_{4}=6.99 \times 10^{-5}$, which is indeed equal to the difference between energies at $c_{1}$ and $c_{2}$ in Fig. 12(b), representing the energy barrier for a jump out of the stable state $c_{1 b}$ via the mountain pass state $c_{2 c}$. In these jumps the pipeline snaps from a localised state with approximately three central periods into a state with approximately four central periods. Similarly, the areas separated by $P_{\mathrm{c} 1}$ are found to be $A_{1}=7.00 \times 10^{-5}$ and $A_{2}=6.96 \times 10^{-5}$. The $P$ values $\left(P_{\mathrm{c} 1}, P_{\mathrm{c} 2}, \ldots\right)$ along successive wiggles where these jumps may happen are very close to the Maxwell load $P_{M}$ and converge (from the right) to $P_{M}$ as we go up the tower.

Under quiet environmental conditions the pipeline will remain in the straight configuration for loads $P>P_{M}$. Fig. 13 shows the energy landscape for successive jumps, indicated in Fig. 12(a), at $P=2.4 \mathrm{MN}$. Localised equilibrium solutions are indicated by black dots. Labels $m_{2}, m_{4}, m_{6}$ and $m_{8}$ represent stable equilibria (energy minima), while $m_{1}, m_{3}, m_{5}$ and $m_{7}$ represent unstable equilibria (here represented by energy maxima). The solid curve connecting these equilibria is not computed directly but, for the purpose of illustration, interpolated by means of a cubic spline. Equilibrium $m_{1}$ (with $E_{d}=$ $1.51847 \times 10^{-4}$ ) represents the mountain pass for the trivial equilibrium (with $E_{d}=0$ ) to escape, possibly to equilibrium $m_{2}$, with one period in the localisation. From this state the pipeline can escape to $m_{4}$ at considerably lower energy cost via $m_{3}$ (or $m_{1}$ ). From $m_{4}$ an even smaller energy hump needs to be overcome to snap into a localised state with more periods and correspondingly higher end shortening $u_{2}$, etc. Hence, once the small energy barrier of equilibrium $m_{1}$ is traversed, the pipeline easily cascades down the energy landscape into localised states with more and more periods. Similar sequential jumping scenarios can be sketched for all loads $P$ between $P_{M}$ and $P_{2}(\approx 2.7)$, while the straight pipeline is globally stable and resistant to small external disturbances for larger $P$ (up to $P_{c r}$ ).

We conclude that under dead loading, with control of $P$, the Maxwell load $P_{M}$ is the lowest load at which another (postbuckled) solution acquires lower energy than the trivial solution. At $P_{M}$ the pipeline only needs to be excited to the energy level of the unstable saddle-type solution with the lowest energy (assuming it is accessible by a well-placed kick) to be forced out of its stable straight state. We call this the mountain pass state for the trivial solution. Looking at Fig. 12(b) (left inset) this 
is seen to be the solution labelled $c_{2 a}$ on branch ' 1 ' (indicated by the big black dot in the figure), with a single period in the localisation, giving an energy barrier of $E_{d}=2.9288 \times 10^{-4}$. Once out of its potential well, there is a vast number of stable states of lower energy available to jump into, with the periodic solution $u_{p}$ having the lowest energy. (Which state is jumped into depends on the nature of the perturbation and the dynamical details of the system, which are outside the present statics theory.) It is therefore safe to assume that under a perturbation imparting an energy $E_{d}=2.9288 \times 10^{-4}$ to the pipeline, it will snap into a localised state taken up almost entirely by a periodic pattern of bounces between the trench walls.

The Maxwell load therefore signals the onset of a super-sensitivity to static and dynamic lateral disturbances. The energy differences between trivial and non-trivial solutions in Fig. 12(b), beyond the Maxwell load, can be interpreted as measures of shock sensitivity of the pipeline (Thompson and van der Heijden, 2014). In practice the required perturbations could for instance be caused by dynamic disturbances due to irregular fluid flow through the pipe, earthquakes or landslides. If periodic bouncing inside the trench is undesirable then the Maxwell load can be considered to be the failure load.

\subsection{Rigid loading ( $u_{2}$ control)}

Here we consider the other extreme case of rigid loading in which the end shortening $u_{2}$ rather than the compressive load $P$ is controlled. The total potential energy density is then given by

$$
E_{r}=E_{b}+E_{f}=\frac{1}{L} \int_{0}^{L}\left(\frac{1}{2} E I w_{3}^{2}+\int_{0}^{w_{1}} F(\mathrm{y}) \mathrm{dy}\right) \mathrm{d} x
$$

In Fig. 14(b) the energy $E_{r}$ is plotted against $u_{2}$. Successive intersections of solution branches mean that wiggles higher up the curve acquire lower energy and therefore take over global stability as $u_{2}$ is increased. Fig. 14(a) shows the bifurcation diagram (with axes interchanged) with this global stability information under control of $u_{2}$ included. Arrows again indicate possible jumps, now at constant $u_{2}$, between equal-energy states along successive stable branches. These jumps occur again at such points that equal areas are cut off, as confirmed by the direct numerical estimates $A_{5}=0.01421, A_{6}=0.01418$ for the two coloured areas in Fig. 14(a) representing the energy barrier for the jump. In the jump from states $t_{3}$ to $t_{4}$ the pipeline develops another period under a dropping axial force $P$. Due to the increasingly slanted nature of the wiggles as $u_{2}$ is increased, the globally stable solution asymptotically approaches the periodic state on $u_{p}$ exactly held at the Maxwell load $P_{M}$.

For the jump $t_{1}-t_{2}$ the energy analysis in Fig. 15 reveals that the bending energy $E_{b}$ goes down, while the foundation energy $E_{f}$ goes up. Meanwhile, the maximum deflection of the pipe in the trench, $w_{m}$, goes down a little, as seen in Fig. 16, which makes sense as the compression $P$ drops in the jump. Under subsequent increase of $u_{2}$ both $E_{b}$ and $E_{f}$ then increase. Under quiet environmental conditions the pipeline will snap at the folds (e.g., $t_{1 \mathrm{a}}$ ) into a localised state with an extra period. Note though that, since both these downward jumps represent reversals of the lateral deformation, the states at the end of the jumps cannot be predicted from the bifurcation diagram.

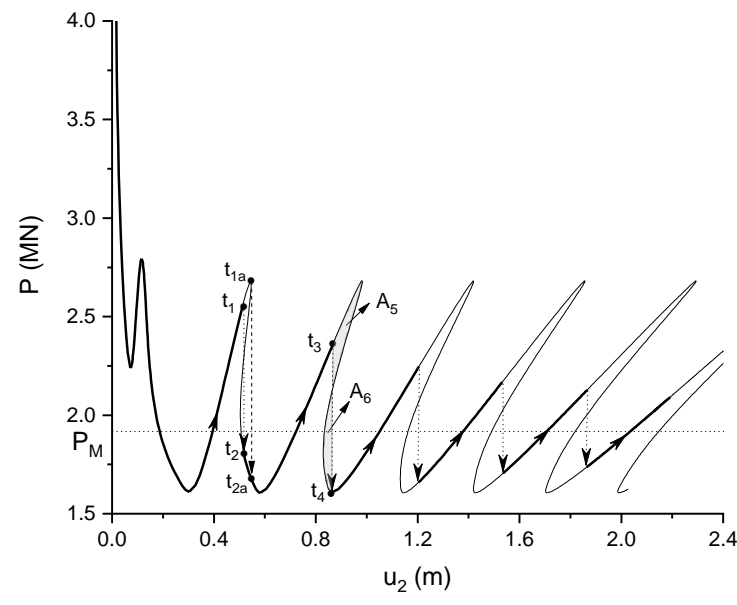

(a)

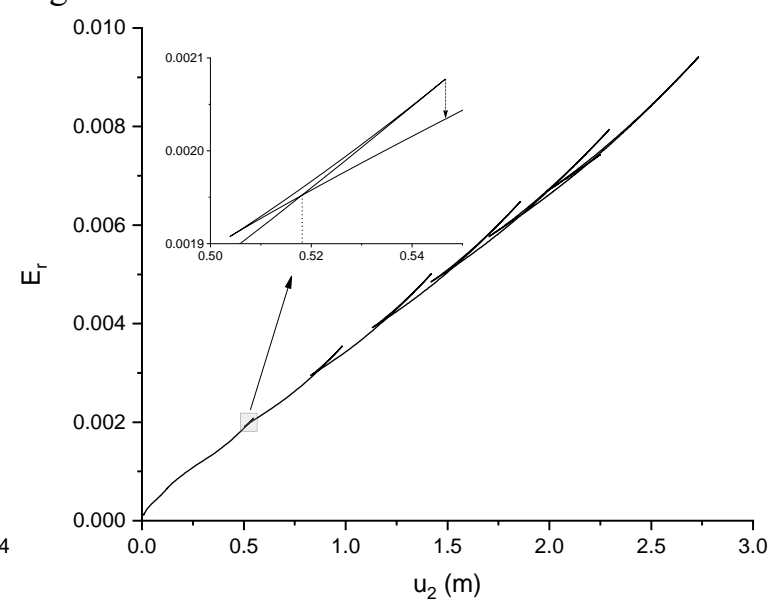

(b)

Fig. 14 Stability analysis for the symmetric homoclinic orbit under rigid loading conditions. (a) Bifurcation diagram. (b)

Total potential energy. $\left(d=3 \mathrm{D}, k=2, \mu_{\text {brk }}=2.1\right.$. $)$ 


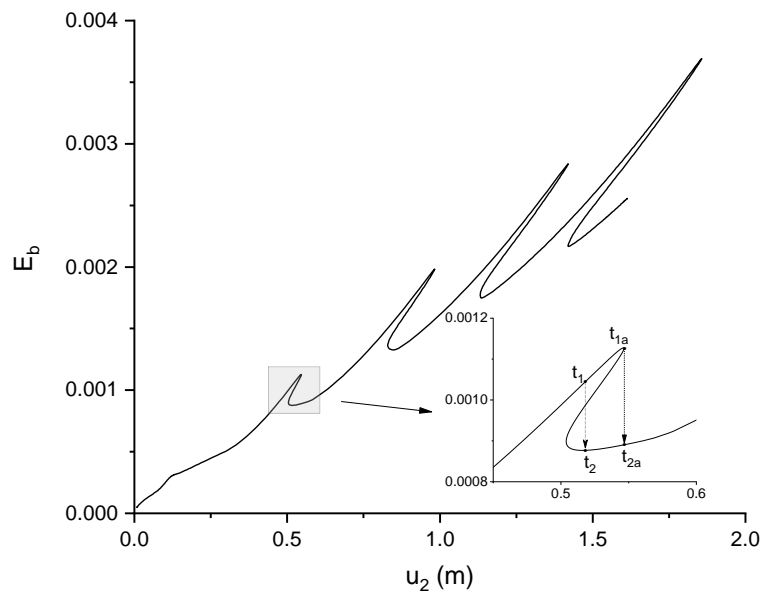

(a)

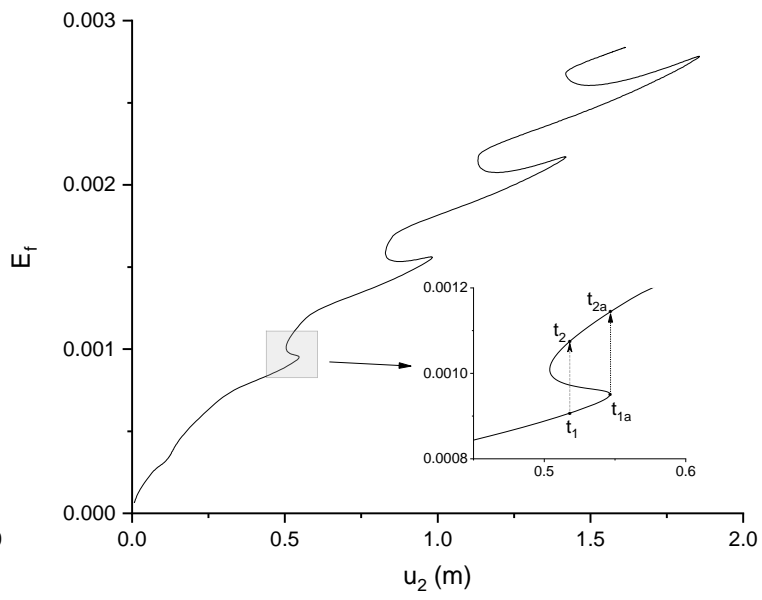

(b)

Fig. 15 Energy analysis under rigid loading conditions. (a) Bending energy $E_{b}$. (b) Foundation energy $E_{f}$. $(d=3 \mathrm{D}, k=2$, $\mu_{b r k}=2.1$.)

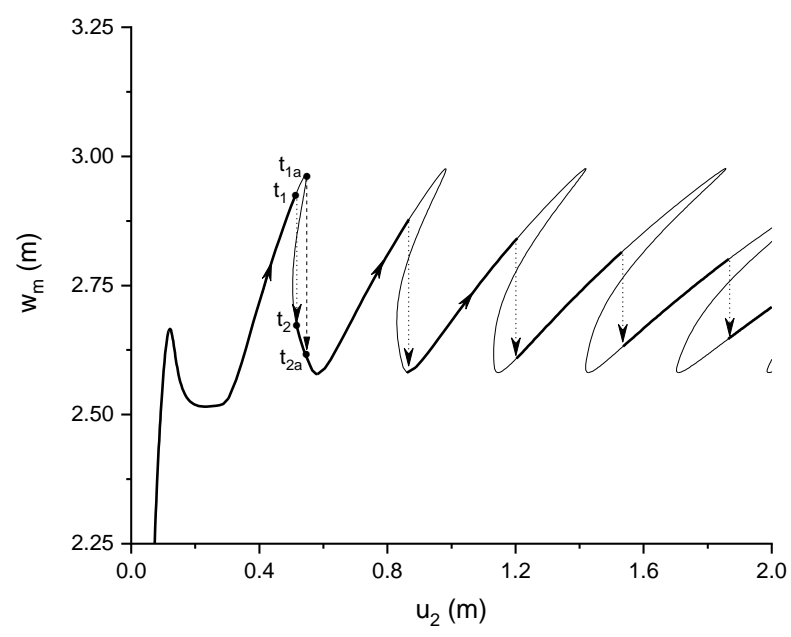

Fig. 16 Relationship between end shortening $u_{2}$ and maximum displacement $w_{m}$ for the symmetric localised solution under rigid loading conditions. $\left(d=3 \mathrm{D}, k=2, \mu_{\text {brk }}=2.1\right.$.)

\subsection{Thermal loading $\left(T_{0}\right.$ control)}

Finally we consider thermal loading by introducing the effect of axial resistance $f_{A}$. Eq. (16) gives the relationship between the temperature difference $T_{0}$ and the axial compressive force $P$. This relationship is depicted for both the symmetric and anti-symmetric localised solutions in Fig. 17. $T_{c r}$ is the critical temperature difference at bifurcation corresponding to the critical axial force $P_{c r}$. We see that at much lower temperature, around $T_{0}=50{ }^{\circ} \mathrm{C}$, localised buckling modes first become available. We confirm that for all solutions, except those close to $T_{c r}, l_{s}>L$, as required for consistency (this makes sense as we recall from Eq. (15) that $l_{s}$ increases with the end shortening $u_{2}$, which goes up as we climb the homoclinic tower).

Fig. 18 gives a zoomed view of the bifurcation diagram for the symmetric homoclinic orbit and also shows the maximum deflection $w_{m}$ and the axial thermal expansion $u_{1}$ as a function of temperature $T_{0}$. The minimum temperature where localised solutions are available is labelled $T_{m}$. Hysteresis cycles (for instance, $b_{2} \rightarrow e_{2} \rightarrow c_{2} \rightarrow e_{3}$ ) with snap behaviour at folds are also indicated but, as commented in Section 3, these have limited quantitative value unless we can interpret our soil resistance law as purely elastic (note that this now applies to both the lateral and the axial resistance). We see, for instance, that in the jump at $b_{2}$ an extra period is created in the pipeline and the axial deformation $u_{1}$ correspondingly goes up but $w_{m}$ goes down slightly in a reversal of lateral deformation. In the presence of imperfections, $T_{m}$ may be considered the maximum safe operating temperature difference of the pipeline (Wang and van der Heijden, 2017). 


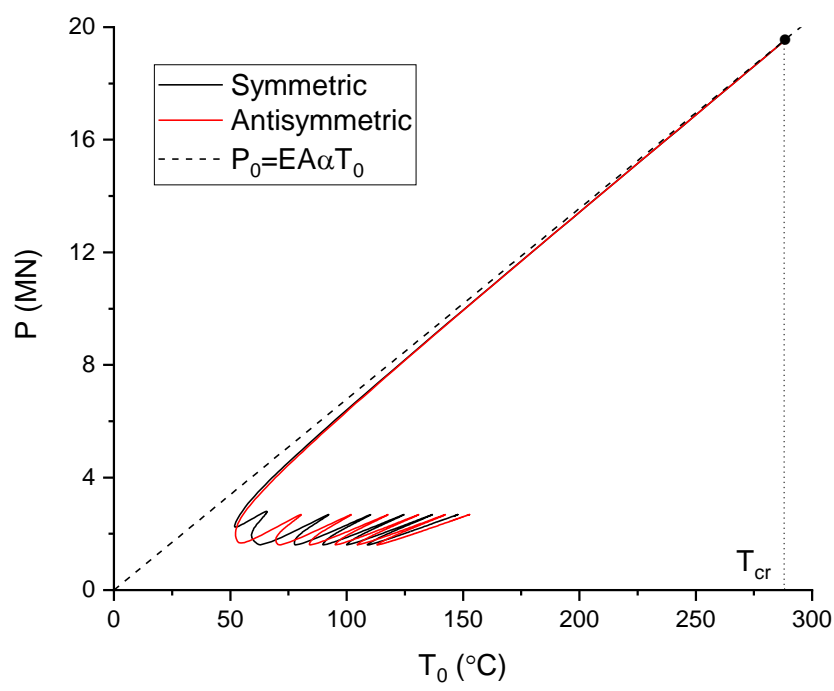

Fig. 17 Relationship between the temperature difference $T_{0}$ and the central axial force $P$ for the localised buckling modes. $\left(d=3 D, k=2, \mu_{b r k}=2.1\right.$.)

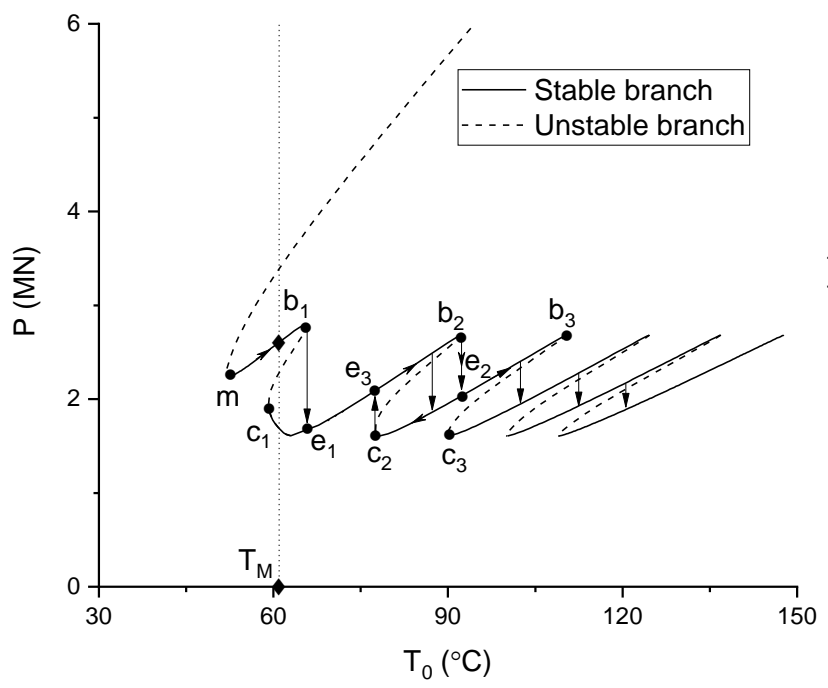

(a)

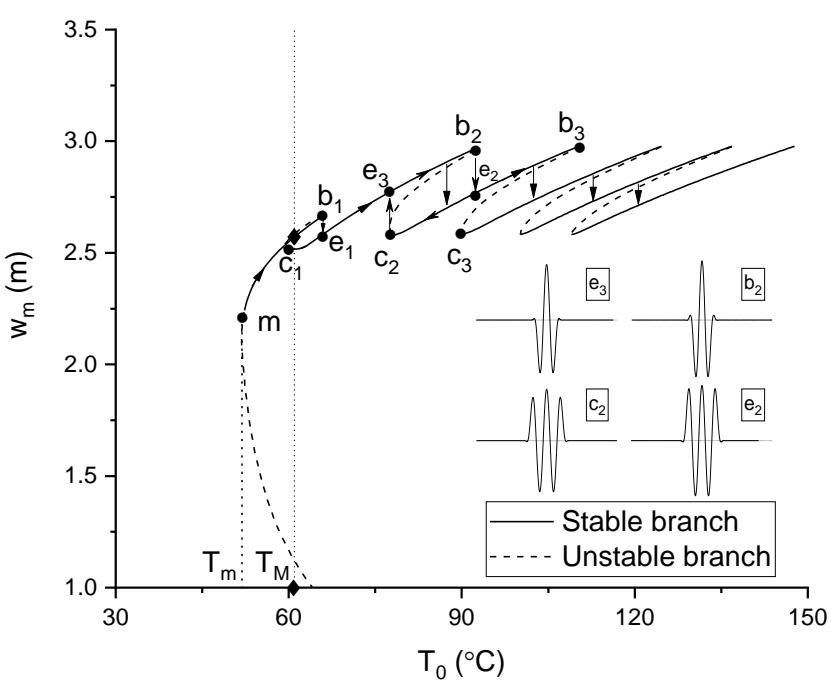

(b)

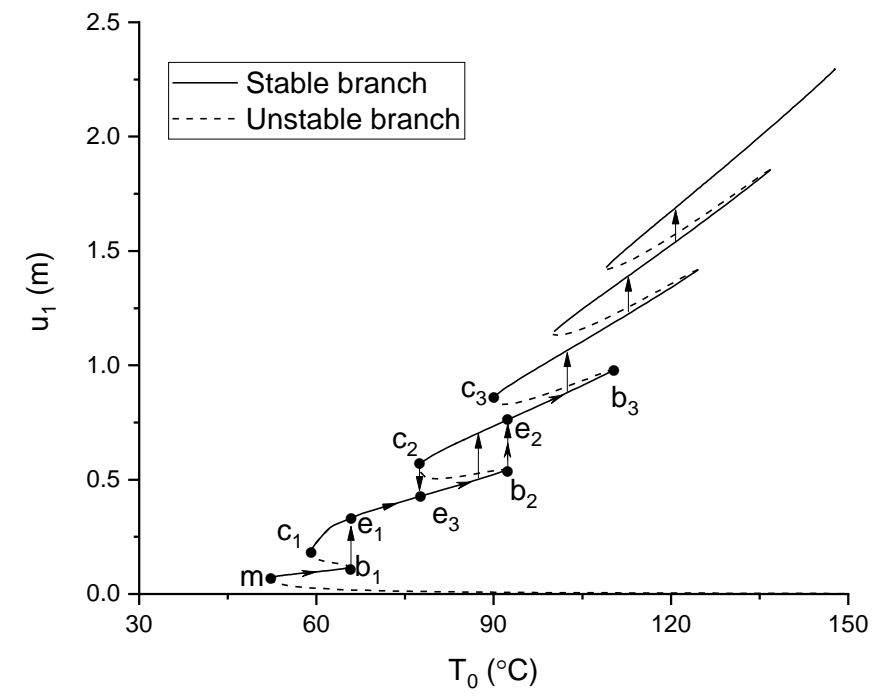

(c)

Fig. 18 Stability of typical buckling behaviour for symmetric localisation. (a) Axial compressive force $P$. (b) Maximum deflection $w_{m}$. (c) Axial thermal expansion $u_{1} .\left(d=3 D, k=2, \mu_{b r k}=2.1.\right)$ 


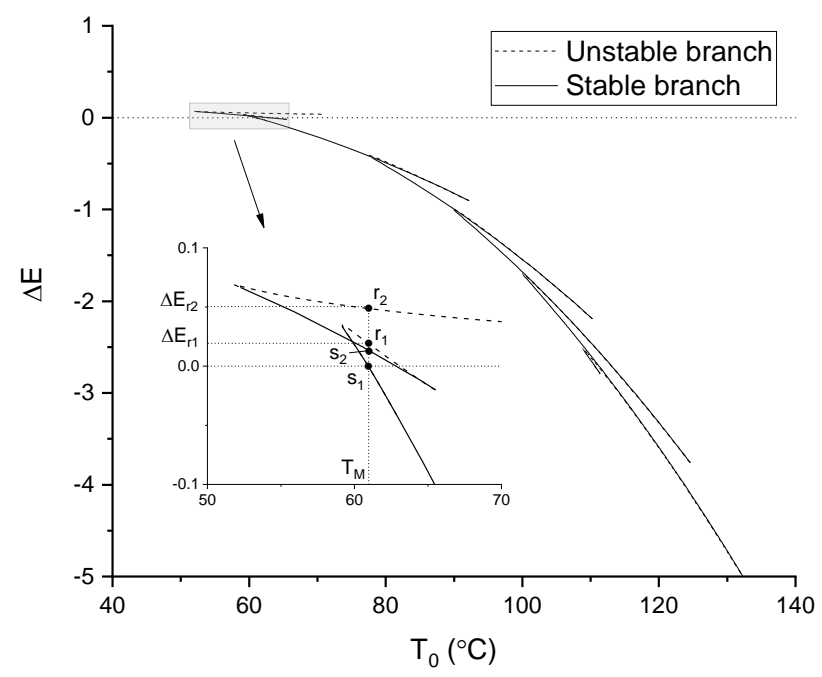

(a)

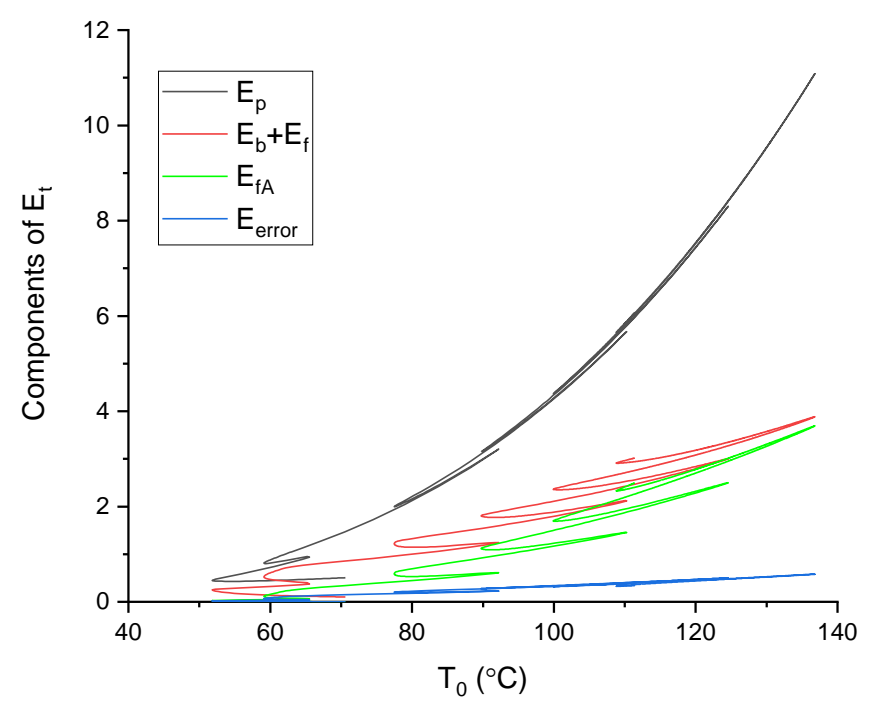

(b)

Fig. 19 Energy analysis under thermal loading. (a) $\Delta E=E_{t}-E_{i}$. (b) The various components of the total energy $E_{t}$. $(d=$ $3 D, k=2, \mu_{b r k}=2.1$.)

Stable solution branches without reversals in either $w$ or $u$ are physical and may be subjected to an energy analysis as in the dead and rigid loading cases. The total potential energy of the straight pipeline, namely before buckling, is now nonzero and given by

$$
E_{i}=\frac{1}{2 E A} \int_{0}^{l_{s}} P_{0}^{2} \mathrm{~d} x
$$

representing the thermal strain energy, while the energy of the buckled pipeline can be written as

$$
E_{t}=E_{b}+E_{f}+E_{p}+E_{f A}=\int_{0}^{L}\left(\frac{1}{2} E I w_{3}^{2}\right) \mathrm{d} x+\int_{0}^{L}\left(\int_{0}^{w_{1}} F(y) \mathrm{d} y\right) \mathrm{d} x+\frac{1}{2 E A} \int_{0}^{l_{s}} \bar{P}^{2} \mathrm{~d} x-\int_{0}^{l_{s}} f_{A} u \mathrm{~d} x
$$

where the first term, $E_{b}$, is the bending energy, the second term, $E_{f}$, is the foundation energy, the third term, $E_{p}$, is the axial strain energy induced by the distributed axial force and the fourth term, $E_{f A}$, is the energy loss due to axial friction. Fig. 19(a) shows the energy difference $\Delta E=E_{t}-E_{i}$ as a function of $T_{0}$ for the branch of symmetric localised solutions. Selfintersections of this curve again correspond to equal-energy states and possible jumps between such equal-energy states are indicated by arrows in Fig. 18.

For nonzero $f_{A}$ a truly periodic solution is unrealistic because it would have an infinite feed-in length $l_{\mathrm{s}}$ and thus, according to Eq. (6), require an infinite pressure difference $P_{0}-P$. It would also have infinite end shortening $u_{2}$ and, by Eq. (16), require an infinite temperature $T_{0}$. We could consider a finite number, $n$, of periods of this solution but we would have a different curve for each $n$ in Fig. 18. Besides, such solutions would only be artificial, non-smooth, localised solutions, pointless because we already have the real localised solutions. We therefore ignore periodic solutions and define the Maxwell temperature $T_{M}$ as the lowest temperature $T_{0}$ where a localised solution has the same energy as the trivial solution. In the limit $f_{A}=0$ this temperature corresponds to the Maxwell load $P_{M}$ as given by Eq. (1). In Fig. 19(a) the Maxwell temperature occurs at $T_{M}=60.9667^{\circ} \mathrm{C}$.

Fig. 19(b) gives the contribution from each of the separate energy sources to the total energy $E_{t}$. Also included in this figure is a plot of $E_{\text {error }}$, defined as the axial potential energy we effectively ignore in the coupled system Eq. (3) by taking $f_{A}=0$ when computing the deflection $w$ in the buckling region:

$$
E_{\text {error }}=-\int_{0}^{L} f_{A} u \mathrm{~d} x=-\int_{0}^{L} f_{A}\left(f_{A} \frac{2 l_{\mathrm{s}} x-x^{2}}{2 E A}-\frac{1}{2} \int_{0}^{x} w_{2}^{2} \mathrm{~d} x\right) \mathrm{d} x
$$

This energy depends on $L$, which is somewhat arbitrary. However, if we take $L=300 \mathrm{~m}$, which is long enough to cover the buckling region for all solutions along the branches shown in Fig. 19(b), then $E_{\text {error }}$ is never more than $6 \%$ of the total energy $E_{t}$. The fact that $E_{\text {error }}$ is significantly smaller than the other energy components is further justification for the approximation to ignore $f_{A}$ in the buckling region. 


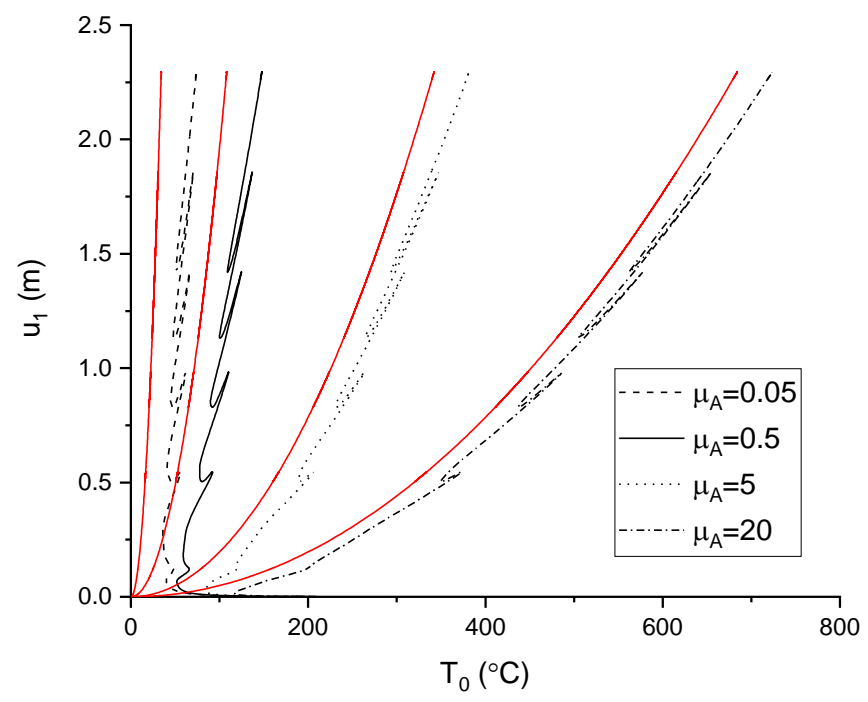

(a)

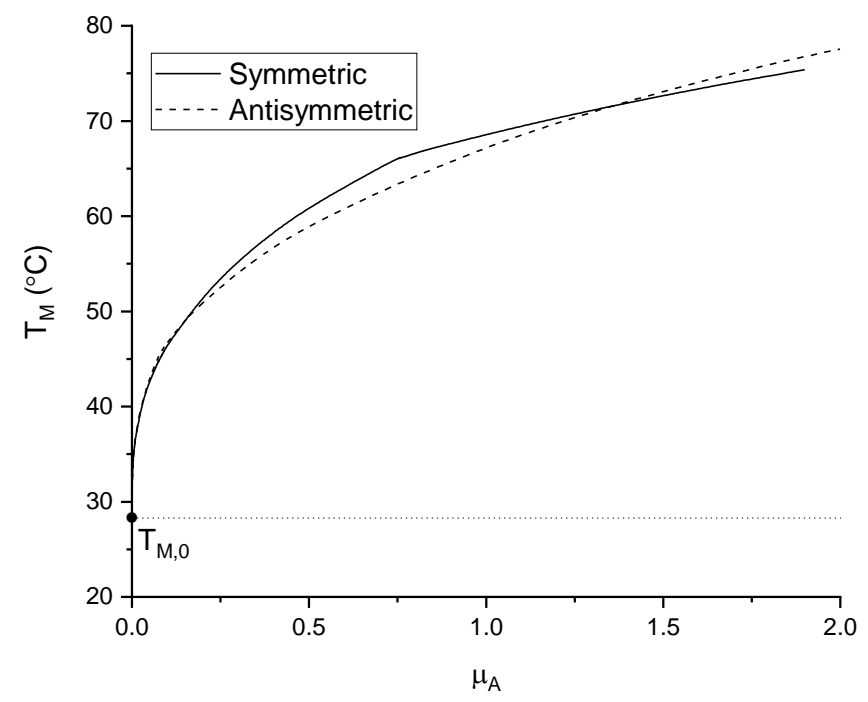

(b)

Fig. 20 (a) Axial thermal expansion $u_{1}$ for different axial friction coefficients $\mu_{A}$. The parabolic red curves represent the rigid-loading limit given by Eq. (16) with $P$ set to zero. (b) Maxwell temperature $T_{M}$ as a function of $\mu_{A}(d=3 D, k=$

$$
\text { 2, } \mu_{\text {brk }}=2.1 \text {.) }
$$

Fig. 20(a) shows the effect of the axial friction coefficient $\mu_{A}$ on the homoclinic tower. Recall that for $\mu_{A}=0$ thermal loading is equivalent to dead loading with the relationship between the compressive load $P$ and temperature $T_{0}$ simply given by Eq. (1). We see in Fig. 20(a) that under increasing $\mu_{A}$ the tower shears to the right. As a result, the Maxwell temperature increases and the bifurcation diagram increasingly comes to resemble the diagram of the rigid-loading case. This is consistent with Eq. (16), which shows that for large $\mu_{A}$ (and hence large $f_{A}$ ), controlling $T_{0}$ becomes equivalent to controlling the end shortening $u_{2}$ (which is equal to the axial expansion $u_{1}$ ). For comparison, the red curves in Fig. 20(a) give the relationship according to Eq. (16) with $P$ set to zero, confirming that this rigid-loading limit is approached for large $\mu_{A}$ (bear in mind here that the difference between red and black curves should be measured vertically). Fig. 20(b) shows the variation of the Maxwell temperature with $\mu_{A}$ (for both symmetric and anti-symmetric localised solutions). It is seen that $T_{M}$ varies extremely sensitively at small values of $\mu_{A}$. The limiting value $T_{M, 0}=28.2852{ }^{\circ} \mathrm{C}$ at $\mu_{A}=0$ corresponds to the Maxwell load $P_{M}=1.9181 \mathrm{MN}$ under dead loading (see Fig. 12), as given by Eq. (1).

As a result of this shearing effect, at given $T_{0}$ fewer localised solutions (stable or unstable) become available as $\mu_{A}$ increases, i.e., shock sensitivity decreases with increasing $\mu_{A}$. One could say that the system becomes less chaotic near the Maxwell temperature $T_{M}$ : jumps become more predictable and under increasing temperature $T_{0}$ we are more likely, for larger $\mu_{A}$, to have successive jumping into a gradually expanding periodic phase. A quantitative expression of this behaviour is given in Fig. 21, where the mountain pass energy $\Delta E_{r}$ is plotted as a function of $\mu_{A}$. This $\Delta E_{r}$ is the energy barrier at $T_{M}$ for escape from the pre-buckled solution via the lowest-energy unstable solution, labelled $r_{1}$ in Fig. 19(a), to the stable solution labelled $s_{1}$ (or the stable solution labelled $s_{2}$ of slightly higher energy, but still lower than that of $r_{1}$ ). Results are given for both symmetric and anti-symmetric localised solutions showing that escape via a symmetric state (induced, most likely, by a symmetric perturbation) generally requires less energy, but for small $\mu_{A}$ the energy landscape becomes very complicated and for small windows of $\mu_{A}$ values anti-symmetric solutions offer lower-energy escape.

For symmetric solutions, we see in Fig. 21(a) that for $\mu_{A}$ larger than about 0.76 the only unstable localised state available is a single-pulse solution, shown in the figure, so escape will necessarily take place via this state. Note that the (also unique) stable state the pipeline jumps into, also shown in the figure, has the same shape as this unstable state but larger amplitude, reaching the trench wall. It is seen that to induce a jump into wall contact it is sufficient, at this $\mu_{A}$ value of about 1.1, to perturb the pipeline a distance of $0.9898 \mathrm{~m}$ or $51 \%$ of the trench width. For $\mu_{A}$ smaller than 0.76 a second unstable solution appears, initially with much lower energy barrier. This solution has a wider pulse with side lobes in the opposite direction, which grow in the final stable state to create three point contacts with the trench wall. The first unstable branch is continued as a dashed curve in this region because it may in practice still be the more likely escape route. For even smaller $\mu_{A}$ gradually 
more escape routes, with more and more periods, become available as we approach the chaotic dead-loading limit where the precise post-buckling state depends sensitively on the value of $\mu_{A}$ (and the precise nature of the perturbation). The dashed curve, meanwhile, terminates at $\mu_{A}=0.0048$.

For anti-symmetric solutions, in Fig. 21(b), broadly similar behaviour is observed but the single-pulse unstable solution is the sole escape route for all $\mu_{A}$ larger than 0.16 , and the stable solution shown in the figure the only destination. This unstable solution also remains available for all $\mu_{A}$ values down to zero (dashed curve).

It turns out to be necessary also to include in this energy discussion the non-symmetric solutions along the rungs in Fig. 10. For these solutions the total energy can be written as

$$
E_{t}=\int_{-L_{l}}^{L_{r}}\left(\frac{1}{2} E I w_{3}^{2}\right) \mathrm{d} x+\int_{-L_{l}}^{L_{r}}\left(\int_{0}^{w_{1}} F(y) \mathrm{d} y\right) \mathrm{d} x+\frac{1}{2 E A} \int_{-l_{s}}^{l_{s}} \bar{P}^{2} \mathrm{~d} x+\int_{-l_{s}}^{l_{s}} f_{A}|u| \mathrm{d} x
$$

Here we have chosen the origin of the $x$ axis at the point in the buckle where the axial displacement is zero so that the full localised solution, of length $L$, can be interpreted as combined left and right half solutions, of half-lengths $L_{l}$ and $L_{r}$, on intervals $-L_{l} \leq x \leq 0$ and $0 \leq x \leq L_{r}$, respectively, where $L_{l}+L_{r}=L$ and $L_{l}$ can be computed from the condition

$$
\int_{-L_{l}}^{0} w_{2}^{2} \mathrm{~d} x=\frac{1}{2} \int_{-L_{l}}^{L_{r}} w_{2}^{2} \mathrm{~d} x
$$

Since both half solutions have the same end shortening $u_{2}$, by Eq. (15) they then also have the same slip length $l_{s}$.

Energy barriers at $T_{M}$ for escape via these non-symmetric solutions are given in Fig. 22, together with the barriers offered by symmetric and anti-symmetric solutions. For comparison with the non-symmetric barriers the barriers of half solutions in Fig. 21 have been multiplied by 2. Fig. 22(a) shows that the single-pulse non-symmetric solution in fact offers lower-energy escape than either the symmetric or anti-symmetric solution for $\mu_{A}$ values between 0.76 and 1.53 (labelled '4' in Fig. 22(a)) and also in a narrow region of $\mu_{A}$ values near 0.2 (labelled ' 2 ' ' in Fig. 22(a)). A representative non-symmetric solution is shown in the figure; $R_{1}$ - and $R_{2}$-reflected versions of this solution have the same energy. The top figure in Fig. 22(b), for $\mu_{A}=0.5$, shows an enlargement of Fig. 19(a) with the branch of non-symmetric solutions (the lowest rung in Fig. 10) included. Solutions along higher rungs have energies lower than the energy of the trivial solution and therefore cannot act as mountain pass states for escape from the trivial solution at $T_{M}$. The curve of non-symmetric solutions in Fig. 22(a) terminates at $\mu_{A}=1.53$, where the first-rung non-symmetric solutions acquire negative $\Delta E_{r}$ as well and therefore no longer offer a mountain pass for escape, as illustrated in the bottom figure of Fig. 22(b). We finally mention that (DNV-RP-F114, 2017) gives $0.3<\mu_{A}<1.0$ as the range of axial friction coefficients for non-carbonate soils. Fig. 22(a) predicts that over this range symmetric solutions offer the dominant lowest-energy escape routes.

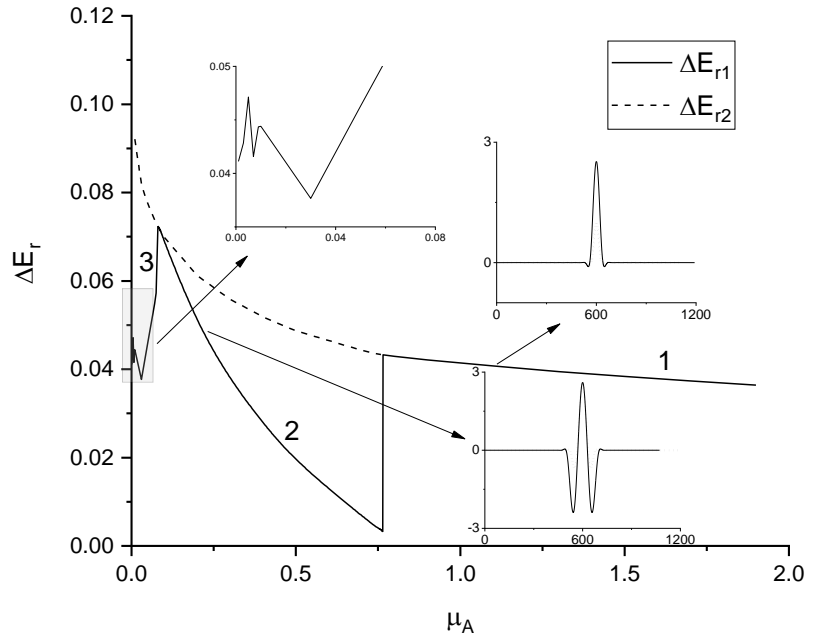

(a)

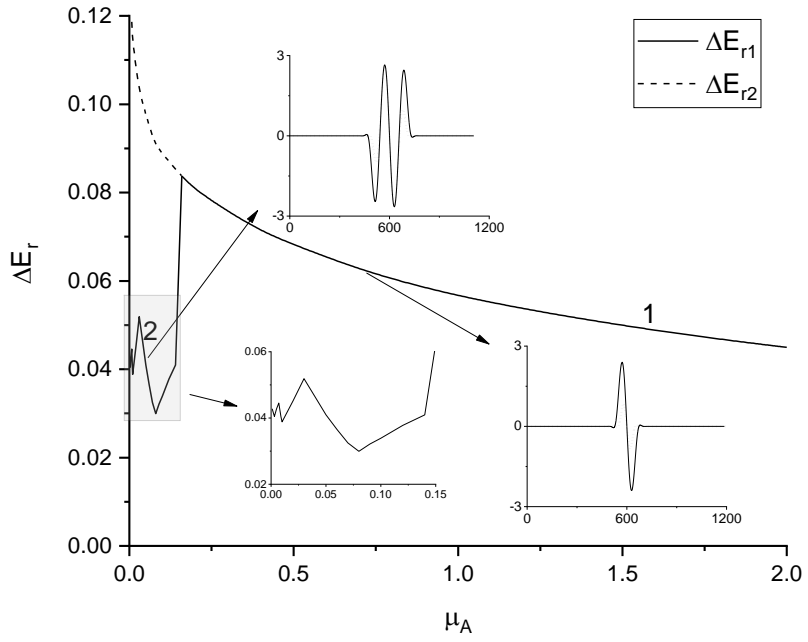

(b)

Fig. 21 Energy barriers at $T_{M}$ for varying axial friction coefficient $\mu_{A}$. (a) Symmetric case. (b) Anti-symmetric case. Integers indicate the number of unstable localised solutions available for escape. Insets show unstable (dashed) and stable (solid) solutions for escape at the indicated points. $\left(d=3 D, k=2, \mu_{b r k}=2.1\right.$.) 


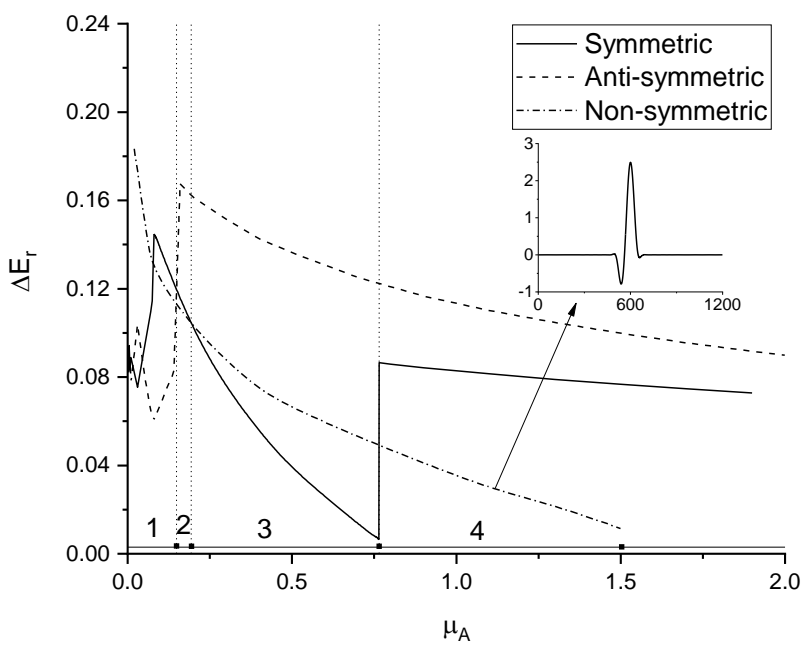

(a)
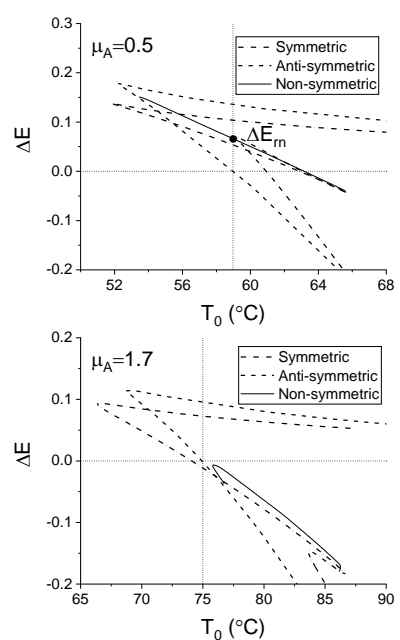

(b)

Fig. 22 (a) Comparison of energy barriers at $T_{M}$ for symmetric, anti-symmetric and non-symmetric solutions. (b) Enlargements of the relevant region near $T_{M}$ in Fig. 19(a). $\left(d=3 D, k=2, \mu_{b r k}=2.1\right.$. $)$

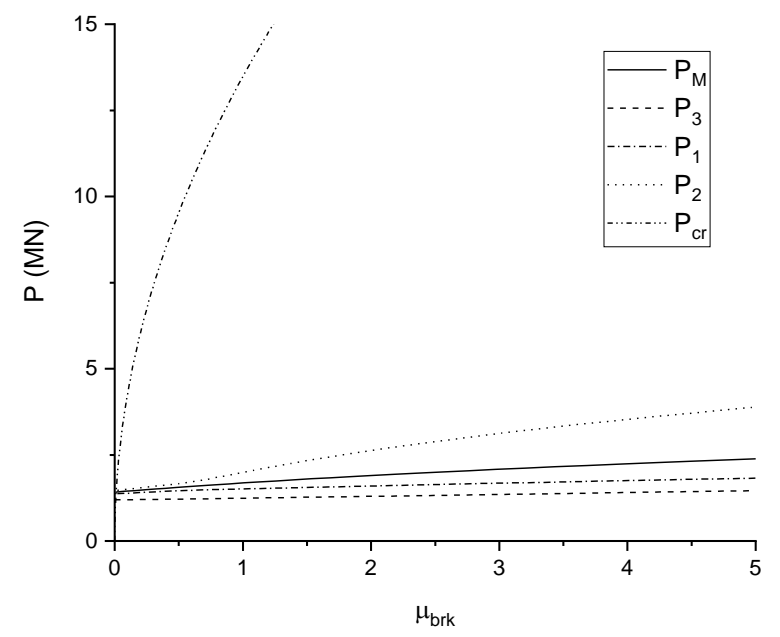

Fig. 23 Effect of $\mu_{r k}$ on the various critical loads.

\section{Effect of varying $\mu_{b r k}$}

In this section we briefly investigate the dependence of the various phenomena observed in the previous sections on the trench parameter $\mu_{b r k}$. This $\mu_{b r k}$, representing the breakout resistance of the soil, is the source of non-monotonicity of the lateral resistance $F$, so one would expect the complicated homoclinic tower to disappear when $\mu_{b r k}$ vanishes and $F$ becomes monotonic. However, our soil resistance model, Eq. (17), is such that even for $\mu_{b r k}=0$ the force $F$ has a nearly horizontal plateau, with exponentially small derivative. As a result, we find that a Maxwell load exists for all values of $\mu_{b r k}$. In the thermodynamic analogy this means that there is no critical point (second-order phase transition) where the curve of first-order phase transitions (coexistence of phases), defined by the Maxwell point, terminates and the two phases become indistinguishable.

In addition, for $\mu_{b r k}=0$ the slope of $F$ at $w=0$ is zero, so $k_{l}=0$ and hence $P_{c r}=0$. The fixed point $j$ is also degenerate as two of its eigenvalues are zero and our shooting approach for computing homoclinic orbits breaks down. We also find that at $\mu_{b r k}=0.275$ the Hamiltonian-Hopf bifurcation changes from being subcritical to being supercritical. This means that for $\mu_{b r k}<0.275$ bifurcating branches of homoclinic and periodic orbits first turn right (towards larger $P$ values) but then make a turn in a fold before they form the homoclinic tower with a Maxwell load larger than the critical load (i.e., $P_{M}>P_{c r}$ ). 
This is confirmed in Fig. 23, which shows the Maxwell load $P_{M}$, as well as the loads $P_{1}, P_{2}$ and $P_{3}$ associated with the homoclinic tower, as a function of $\mu_{b r k}$. The Maxwell load goes down monotonically under decreasing $\mu_{b r k}$. The width of the tower, i.e., the interval $\left[P_{1}, P_{2}\right]$, decreases with $\mu_{b r k}$ but has a finite limit at $\mu_{b r k}=0$.

\section{Summary and discussion}

We have studied localisation in a slender structure resting on a nonlinear foundation that provides both lateral and axial resistance. The lateral resistance is of destiffening-restiffening type known to lead to complex localisation and jump phenomena near a Maxwell critical load. By considering the coupling of lateral and axial deformation we are able to investigate the mitigating effect of axial resistance on these phenomena. To have a concrete and realistic example we consider the case of a partially embedded trenched subsea pipeline but our results hold qualitatively for a wide class of problems with non-monotonic lateral resistance.

The main assumption in our mathematical modelling is that we take the compressive force $\bar{P}$ in the localised buckle to be constant. This assumption is commonly made in the literature and justified by an energy analysis. However, it is good to stress that we make this assumption only in computing the deflection $w$ of the localised solution and not in the treatment of axial deformation which relates the compressive force to temperature and not when computing the energy for the stability analysis. The buckling problem then separates into semi-decoupled lateral and axial deformation problems, the first governed by a fourth-order uniform beam-column equation, the second by a second-order bar equation. Only the axial problem depends on the axial resistance.

The beam-column equation for bending is valid for small deflections from the straight unstressed state of the pipe. This is sufficient in the current application because the trench prevents large deformations. It is the steeply rising stiffness of the trench walls, combined with the initial breakout resistance of the soil, that introduces nonlinearity into the model causing complicated buckling and post-buckling behaviour. The reduced fourth-order description allows us to study this complexity by means of powerful techniques and results from dynamical systems theory valid in a 4D phase space in which the localised solutions are described by homoclinic orbits.

Given the lateral deflection, the axial problem is easily solved and we use deformational compatibility between lateral and axial response within the longer immobilised pipeline to relate the compressive force at the centre of the buckle to the temperature difference $T_{0}$ between pipeline and environment, thereby coupling the lateral and axial system of equations.

We find that linear instability is described by a Hamiltonian-Hopf bifurcation, at a critical load $P_{c r}$ that only depends on the linear stiffness $k_{l}$ of the soil. Nonlinear instability, however, under finite perturbations, our main concern here, is found to be governed by a Maxwell critical load $P_{M}$ that typically occurs at much smaller loads. It is near this load that complicated jump phenomena occur.

Coupling of axial and lateral deformation under thermal loading leads to an interpolation between dead and rigid loading conditions governed by the axial resistance. For zero axial resistance $\left(\mu_{A}=0\right)$ the pipe is effectively under a dead axial load $P$ (given by the temperature difference $T_{0}$ ). Under this condition the pipeline is found to experience shock sensitivity once loaded past the Maxwell load $P_{M}$, where a large number of stable localised solutions become available. It takes only a small perturbation for the stable straight pipe to be forced into a localised post-buckled state. After this initial jump the pipe is then overwhelmingly likely, by only tiny disturbances, to cascade to more extended localised solutions with more and more periods (approaching the periodic phase of repeated bounces between the trench walls). We have identified and quantified the minimal energy barrier, represented by an unstable mountain pass state ( $c_{2 a}$ in Fig. 12), that needs to be overcome for this escape of the straight pipeline from its potential well to a localised state.

For positive axial resistance $\left(\mu_{A}>0\right)$ we define the 'Maxwell temperature' $T_{M}$ to be the lowest temperature where a localised solution acquires the same energy as the pre-buckled straight solution. The effect of increased axial resistance is to push this $T_{M}$ to higher values and to reduce shock sensitivity. This happens through a shearing of the bifurcation diagram (seen in Fig. 20(a)) as a result of which localised solutions are pulled away from the critical point $T_{M}$. Fewer post-buckled states are then available, making the buckling process more predictable. For large axial resistance the pipeline is effectively under rigid loading and is more likely to jump successively into expanding localised states with more and more periods. The 
diminished shock sensitivity under increasing axial resistance is quantified by the energy barrier plots in Fig. 21 and Fig. 22. We find that, depending on the value of the axial resistance coefficient $\mu_{A}$, lowest-energy escape is offered by either a symmetric, an anti-symmetric or a non-symmetric unstable solution (and expectedly induced by disturbances of the same type).

Energy barriers for jumps out of non-trivial solutions, as for instance those at loads $P_{\mathrm{c} 1}$ and $P_{\mathrm{c} 2}$ in Fig. 12(a), could similarly be analysed but are harder to interpret in our present model because our lateral as well as axial (static) resistances are assumed to have a definite sign and therefore are frictional only in the loading phase (not the unloading phase). Energy barriers for jumps between states computed here are thus only valid as long as both lateral and axial deformations proceed monotonically, which, as Fig. 18 shows, need not always be the case for jumps out of non-trivial states. There seems to be no easy a priori characterisation of monotonic deformations, but for jumps that involve only such monotonic deformations our energy analysis, based on Eq. (36), would be valid.

Even under quiet environmental conditions complicated snap behaviour, induced by folds, should be expected near the Maxwell load/temperature, although this behaviour is not described in detail by our present model because we can only physically interpret (quasi-static) loading sequences that avoid reversals in either lateral or axial deformation. Complete hysteresis cycles under thermal loading, as in Fig. 18, are therefore not entirely realistic, but the jumps at the folds are. Hysteresis cycles can therefore be expected (albeit between slightly different states) in cases where the temperature goes up as well as down. This may for instance be caused by periodic pipeline start-ups and shut-downs.

It is good to realise that even if we know which unstable solution offers the easiest escape (in terms of energy) then this need not say much about the likelihood of escape in practice occurring in this way. This practical escape is a dynamics problem and it is hard to predict what type of dynamical perturbation would be required for escape from a particular state. It may, for instance, require a symmetrical perturbation which in practice would be hard to be induced by fluid flow inside the pipe, or would be destroyed by gravity. Once escaped it is also hard to predict in which stable state the system ends up. It is certainly not required that states with one, two, three periods are visited in neat succession; states may be skipped (and even be jumped back into). It would be interesting to do a proper dynamical analysis of this pipeline buckling problem.

\section{Acknowledgments}

ZW acknowledges support by the International Postdoctoral Fellowship Program of the China Postdoctoral Council (No.20180049).

\section{Appendix: Maxwell load, homoclinic snaking and periodic patterns}

The oscillatory bifurcation curves of localised solutions found in this work are reminiscent of the homoclinic snaking observed in the widely studied Swift-Hohenberg equation usually written in the form

$$
\frac{\partial u}{\partial t}=r u-\left(\partial_{x}^{2}+q_{c}^{2}\right)^{2} u+N(u)
$$

where $r$ and $q_{c}$ are parameters, $\partial_{x}$ is the partial derivative with respect to the spatial variable $x$ and $N(u)$ is a nonlinear function usually taken polynomial (Knobloch, 2015). The PDE (A.1) was originally introduced to study the effects of thermal fluctuations on convective hydrodynamic instability (Swift and Hohenberg, 1977), but has since been used extensively as a scalar model to study various types of behaviour associated with pattern formation: convection rolls (or cells) in hydrodynamics, solidification fronts in supercooled liquids, solitary waves in fibre optics, oscillatory chemical reactions, etc. (Cross and Hohenberg, 1993).

For stationary (i.e., time-independent) solutions (patterns) Eq. (A.1) reduces to an ODE exactly of the form of our beamcolumn equation (Eq. (7)). To observe the equilibrium phenomena discussed in Section 3, $N$ has to be non-monotonic, cubiclike, but the precise details are otherwise unimportant (Peletier, 2001). Our $F$, although not polynomial, is of this form thanks to the softening-restiffening characteristic of the combined soil and trench restraint shown in Fig. 3. Similar nonlinearities have been considered in the study of cellular buckling of long structures (Hunt et al., 2000). 
What follows below is a physical and mathematical explanation of the complicated bifurcation behaviour in Fig. 6, which to a large extent is universal and seen in a variety of systems. For the purpose of this discussion it is convenient to think of the compressive force $P$ in the pipe as a control parameter (in (A.1), $r$ is usually taken as the bifurcation parameter). We can then consider the lateral deformation in the central buckled segment of pipe isolated from the axial deformation and thermal effects. Our results are then also more comparable to those of localisation studies in the literature (Hunt et al., 2000; Knobloch, 2015).

It is easy to imagine that a trenched pipeline under a compressive load may buckle into a periodic configuration with the pipe bouncing back and forth between the walls of the trench. This is made possible in the present model by the strongly stiffening characteristic of the restoring force $F$ for relatively large lateral displacements (see Fig. 3). However, starting from a straight pipeline, as we do, we will only observe a transition to this periodic state if the energies of the straight and periodic states are not too widely separated. In our system the two energy densities become in fact equal at a certain critical load $P_{M}$ called the Maxwell load. At that point we have a 'phase transition' from the straight to the periodic configuration.

Indeed, the Maxwell critical point is well-known from first-order phase transitions in thermodynamics and statistical mechanics (for instance, the liquid-gas transition) (Reichl, 2016). At the Maxwell point one can have coexistence of states. Different states (phases) can occur together in equilibrium because they have the same energy density, so there is no tendency for one phase to 'invade' the other. The analogy in our pipeline is that a solution with given total energy can be constructed from arbitrary pieces of straight and periodic solutions put together. The analogy is not complete because in our mechanical problem the pieces would have to be put together subject to continuity conditions (on slope and curvature) to maintain equilibrium, but in the limit of an infinitely long pipe (the 'thermodynamic limit') the energy associated with these transitions would become insignificant compared to the bulk energy and we would have a true first-order phase transition. In the thermodynamic analogy the load-deflection curve in Fig. 6 is an isotherm in a pressure-volume phase diagram.

Now, in thermodynamic systems, phase transitions normally occur at a single sharp value of the control parameter (e.g., pressure) because both phases are homogeneous. If the control parameter is changed the energy densities of the two phases change differently, which means that energy balance is disturbed. The total energy of the system can be reduced by favouring one phase over the other: the phase with lower energy density will 'invade' the phase with higher energy density and ultimately dominate the entire system. In a dynamical context, such as in the Swift-Hohenberg PDE, we would have moving fronts or interfaces between phases. Thus, any small change from the critical parameter value would destroy equilibrium.

In our case, however, one state (the trivial state) is homogeneous, but the other state (the periodic state) is not. It is this inhomogeneity that leads to the complicated snaking behaviour seen in Fig. 6 . The reason is that the periodic state has some flexibility (slack): the period can vary.

Thus, as the load $P$ varies away from the critical value $P_{M}$ the period of the periodic solution varies and hence the energy density varies. A transitory solution between the two states is however still possible because the total energy can be redistributed over slightly different lengths of straight and periodic phases. This mechanism, anticipated by (Pomeau, 1986), is why localised solutions can exist for $P$ away from $P_{M}$ and why the homoclinic snaking is observed. Specifically, in our pipeline problem, for $P<P_{M}$ the straight solution is energetically preferred (see Fig. 6(b)). In the dynamical context (Eq. (A.1)), the front between the two phases would tend to move into the periodic phase. However, this would have to take place against expanding periods in the periodic phase as a result of the relaxed compression $P$. A new equilibrium will be established, which, as Fig. 9 shows, has an increased wavelength in the periodic phase, compared to the wavelength at $P_{M}$. Similarly, for $P>P_{M}$ the periodic solution is energetically preferred, so the front in the coexistent phase (localised solution) would tend to move into the straight phase, but this would have to take place against an increased compression $P$ and the net effect is that the wavelength decreases. (In the Swift-Hohenberg equation, where the 'linear stiffness' $r$ is varied, opposite wavelength behaviour through the Maxwell point is observed: for $r>r_{M}$ the wavelength increases.)

This flexibility to adjust the period is, however, finite, which means that over some interval $\left[P_{1}, P_{2}\right]$ of $P$ values straddling $P_{M}$ we have localised solutions consisting of pieces of straight and periodic parts. The precise width of the interval depends on the details of the system. In the dynamical context, it takes a certain amount of energy, and therefore a finite deviation of $P$ from $P_{M}$, to induce moving fronts because the periodic phase readjusts itself by varying its period. The interface is said, 
in (Pomeau, 1986), to be 'pinned' on the periodic state and the energy is referred to as a 'pinning energy'. In conclusion, the critical Maxwell load is broadened into an interval due to the heterogeneity of the periodic phase.

As we go up the tower in Fig. 6 more and more periods occur in the periodic part (see Fig. 8). Of course we need a sufficiently long pipe to accommodate a large number of periods. At the top of the tower (in an infinitely long pipe, i.e., in the 'thermodynamic limit') the localised solutions approach so-called heteroclinic solutions connecting an infinitely long straight phase with an infinitely long periodic phase. These periodic phases have different periods for different $P$ in $\left[P_{1}, P_{2}\right]$ (cf. Fig. 9). The one at $P_{M}$ has the same energy density as the straight phase and therefore marks the phase transition.

This phase transition is of course only possible if, in addition to the trivial straight state, a periodic state is available. This is perhaps physically plausible as a bouncing solution between the trench walls, but mathematically the periodic orbit has to be of a special type. This is because the system of equations has a conserved quantity, namely the Hamiltonian $H$. This $H$ is thus constant along solutions. Now, since the trivial solution has $H=0$, all localised solutions in the tower will also have $H=0$ because they are asymptotically connected to the trivial solution (the saddle point in the phase space, cf. Fig. 5(b)). But then the required periodic orbit will also have $H=0$ because it is part of the localised solution, high up the tower. It would at first sight seem very special to have a periodic orbit that has exactly $H=0$ at $P=P_{M}$. However, results from dynamical systems theory tell us that we can in fact expect this to happen near a Hamiltonian-Hopf bifurcation, which we have as our central bifurcation at $P=P_{c r}$.

In a Hamiltonian-Hopf bifurcation homoclinic orbits (localised solutions) are generated (Iooss and Pérouème, 1993; Robinson, 1970), as computed in Section 3. Near these homoclinic orbits there exists a two-parameter family of periodic solutions that converge to the homoclinic orbit (Devaney, 1977). This means that we can impose a condition on the periodic orbit and still have a one-parameter curve of periodic solutions coming out of $P_{c r}$. This is exactly the curve $u_{p}$ in Fig. 6 with condition $H=0$ satisfied. Within the two-parameter family of periodic solutions the two conditions $H=0$ and $E_{d}$ (periodic) $=E_{d}$ (straight) pick out a unique solution at a certain critical value $P_{M}$ and with certain wavelength. This is the periodic solution indicated by the black dot in Fig. 6. One might say that the two conditions in our conservative system select the wavelength of the periodic pattern (Nepomnyashchy et al., 1994). Thus it is the multiplicity of periodic orbits near a Hamiltonian-Hopf bifurcation that makes possible the interaction between the curves of localised and periodic solutions and therefore the homoclinic snaking and the broadening of the Maxwell load into an interval $\left[P_{1}, P_{2}\right]$.

Variation of any parameters in the nonlinear function $N(u)$, such as the breakout coefficient $\mu_{b r k}$ in the pipe-soil interaction model in Eq. (17), may remove the non-monotonicity of $N(u)$ (similar to the role played by temperature in the pressure-volume phase diagram in thermodynamics). For a monotonic $N(u)$ a branch of periodic solutions $\left(u_{p}\right)$ will then still emerge from the Hamiltonian-Hopf bifurcation, alongside the branches of localised solutions $\left(u_{s}\right.$ and $\left.u_{a}\right)$. However, the periodic and localised branches no longer interact, in the sense that there is no longer a load $P$ at which both types of solution have the same energy density. No phase transition and no homoclinic snaking occurs. The critical parameter value where nonmonotonicity is lost corresponds to a second-order phase transition in thermodynamics (at critical temperature). It occurs as the end point of a curve of first-order phase transitions (Maxwell loads) in the temperature-pressure phase diagram (parameter space), where the two phases become indistinguishable.

At the level of the underlying phase-space dynamics, it is the chaotic nature of the $4 \mathrm{D}$ system of equations that explains the existence of all the required periodic and homoclinic solutions. More precisely, this complex (horseshoe) dynamics exists due to so-called heteroclinic tangles (Woods and Champneys, 1999). These exist for parameter values between two critical values corresponding to tangencies of invariant manifolds of the trivial solution (fixed point at the origin) and the periodic orbit (Coullet et al., 2000; Knobloch, 2015; Woods and Champneys, 1999). These critical parameter values are precisely the $P_{1}$ and $P_{2}$ in Fig. 6. In between these values we have 'spatial chaos', here manifested by the infinitely many localised solutions in the homoclinic tower. This interval $\left[P_{1}, P_{2}\right]$, therefore, is the asymptotic interval at the top of the tower (in the 'thermodynamic limit') where heteroclinic connections between the trivial solution and a periodic solution exist. The oscillations lower down in the tower slightly move away from the interval $\left[P_{1}, P_{2}\right]$ due to finite-size effects.

For dimensional reasons the above scenario holds generally in a 4D system: if the system is chaotic then we have a 'smeared-out' Maxwell load, i.e., the interval $\left[P_{1}, P_{2}\right]$; if the system is not chaotic then a phase transition can still occur but 
it will occur at a sharply defined Maxwell load $P_{M}$ (and involve a homogeneous non-trivial state, namely a fixed point). A particularly transparent example of a system in which, depending on parameters, either a sharp critical Maxwell load $P_{M}$ occurs or a critical interval $\left[P_{1}, P_{2}\right]$ is that of a rod constrained to lie on a cylinder (van der Heijden, 2001; van der Heijden et al., 2002).

\section{References}

Avitabile, D., Lloyd, D.J., Burke, J., Knobloch, E., Sandstede, B., 2010. To snake or not to snake in the planar Swift-Hohenberg equation. SIAM Journal on Applied Dynamical Systems 9, 704-733.

Burke, J., Knobloch, E., 2006. Localized states in the generalized Swift-Hohenberg equation. Physical Review E 73, 056211.

Burke, J., Knobloch, E., 2007. Snakes and ladders: Localized states in the Swift-Hohenberg equation. Physics Letters A 360, 681-688.

Champneys, A.R., Spence, A., 1993. Hunting for homoclinic orbits in reversible systems: a shooting technique. Advances in Computational Mathematics 1, 81-108.

Chatterjee, S., White, D.J., Randolph, M.F., 2012. Numerical simulations of pipe-soil interaction during large lateral movements on clay. Géotechnique 62, 693-705.

Coullet, P., Riera, C., Tresser, C., 2000. Stable static localized structures in one dimension. Physical Review Letters 84, 3069.

Cross, M.C., Hohenberg, P.C., 1993. Pattern formation outside of equilibrium. Reviews of Modern Physics 65, 851.

Devaney, R.L., 1977. Blue sky catastrophes in reversible and Hamiltonian systems. Indiana University Mathematics Journal 26, 247-263.

DNV-RP-F109, 2011. On-bottom stability design of submarine pipelines, RP-F109. Oslo: Det Norske Veritas.

DNV-RP-F110, 2018. Global buckling of submarine pipelines structural design due to high temperature/high pressure. Oslo: Det Norske Veritas.

DNV-RP-F114, 2017. Pipe-soil interaction for submarine pipelines. Oslo: Det Norske Veritas.

Groh, R.M.J., Pirrera, A., 2019. On the role of localizations in buckling of axially compressed cylinders. Proceedings of the Royal Society A 475, 20190006.

Hobbs, B.E., Ord, A., 2012. Localized and chaotic folding: the role of axial plane structures. Philosophical Transactions of the Royal Society A 370, 1966-2009.

Hobbs, R.E., 1984. In-service buckling of heated pipelines. Journal of Transportation Engineering 110, 175-189.

Hong, Z., Liu, R., Liu, W., Yan, S., 2015. Study on lateral buckling characteristics of a submarine pipeline with a single arch symmetric initial imperfection. Ocean Engineering 108, 21-32.

Hunt, G.W., Bolt, H., Thompson, J., 1989. Structural localization phenomena and the dynamical phase-space analogy. Proceedings of the Royal Society A 425, 245-267.

Hunt, G.W., Lord, G., Champneys, A., 1999. Homoclinic and heteroclinic orbits underlying the post-buckling of axiallycompressed cylindrical shells. Computer Methods in Applied Mechanics and Engineering 170, 239-251.

Hunt, G.W., Peletier, M.A., Champneys, A.R., Woods, P.D., Ahmer Wadee, M., Budd, C.J., Lord, G.J., 2000. Cellular buckling in long structures. Nonlinear Dynamics 21, 3-29.

Iooss, G., Pérouème, M.-C., 1993. Perturbed homoclinic solutions in reversible 1: 1 resonance vector fields. Journal of Differential Equations 102, 62-88.

Jin, L., Takei, A., Hutchinson, J.W., 2015. Mechanics of wrinkle/ridge transitions in thin film/substrate systems. Journal of the Mechanics and Physics of Solids 81, 22-40.

Kerr, A.D., 1978. Analysis of thermal track buckling in the lateral plane. Acta Mechanica 30, 17-50.

Knobloch, E., 2015. Spatial localization in dissipative systems. Annual Review of Condensed Matter Physics 6, 325-359.

Knobloch, J., Lloyd, D.J., Sandstede, B., Wagenknecht, T., 2011. Isolas of 2-pulse solutions in homoclinic snaking scenarios. Journal of Dynamics and Differential Equations 23, 93-114. 
Nepomnyashchy, A.A., Tribelsky, M.I., Velarde, M.G., 1994. Wave number selection in convection and related problems. Physical Review E 50, 1194.

Peletier, M.A., 2001. Sequential buckling: A variational analysis. SIAM Journal on Mathematical Analysis 32, $1142-1168$.

Pocivavsek, L., Dellsy, R., Kern, A., Johnson, S., Lin, B., Lee, K.Y.C., Cerda, E., 2008. Stress and fold localization in thin elastic membranes. Science 320, 912-916.

Pomeau, Y., 1986. Front motion, metastability and subcritical bifurcations in hydrodynamics. Physica D 23, 3-11.

Reichl, L.E., 2016. A Modern Course in Statistical Physics (4th ed.). Wiley-VCH.

Robinson, R.C., 1970. Generic properties of conservative systems. American Journal of Mathematics 92, $562-603$.

Swift, J., Hohenberg, P.C., 1977. Hydrodynamic fluctuations at the convective instability. Physical Review A 15, 319.

Taylor, N., Gan, A.B., 1986a. Refined modelling for the lateral buckling of submarine pipelines. Journal of Constructional Steel Research 6, 143-162.

Taylor, N., Gan, A.B., 1986b. Submarine pipeline buckling-imperfection studies. Thin-Walled Structures 4, $295-323$.

Thompson, J.M.T., van der Heijden, G.H.M., 2014. Quantified "shock-sensitivity" above the Maxwell load. International Journal of Bifurcation and Chaos 24, 14.

van der Heijden, G.H.M., 2001. The static deformation of a twisted elastic rod constrained to lie on a cylinder. Proceedings of the Royal Society A 457, 695-715.

van der Heijden, G.H.M., Champneys, A.R., Thompson, J.M.T., 1998. The spatial complexity of localized buckling in rods with noncircular cross section. SIAM Journal on Applied Mathematics 59, 198-221.

van der Heijden, G.H.M., Champneys, A.R., Thompson, J.M.T., 2002. Spatially complex localisation in twisted elastic rods constrained to a cylinder. International Journal of Solids and Structures 39, 1863-1883.

Wang, Z., Tang, Y., Feng, H., Zhao, Z., Liu, H., 2017. Model test for lateral soil resistance of partially embedded subsea pipelines on sand during large-amplitude lateral movement. Journal of Coastal Research 33, 607-618.

Wang, Z., Tang, Y., van der Heijden, G.H.M., 2018. Analytical study of lateral thermal buckling for subsea pipelines with sleeper. Thin-Walled Structures 122, 17-29.

Wang, Z., van der Heijden, G.H.M., 2017. Localised lateral buckling of partially embedded subsea pipelines with nonlinear soil resistance. Thin-Walled Structures 120, 408-420.

White, D.J., Cheuk, C.Y., 2008. Modelling the soil resistance on seabed pipelines during large cycles of lateral movement. Marine Structures 21, 59-79.

Woods, P.D., Champneys, A.R., 1999. Heteroclinic tangles and homoclinic snaking in the unfolding of a degenerate reversible Hamiltonian-Hopf bifurcation. Physica D 129, 147-170.

Zeng, X., Duan, M., 2014. Mode localization in lateral buckling of partially embedded submarine pipelines. International Journal of Solids and Structures 51, 1991-1999.

Zhang, X., Guedes Soares, C., 2019. Lateral buckling analysis of subsea pipelines on nonlinear foundation. Ocean Engineering $186,106085$.

Zhu, J., Attard, M.M., Kellermann, D.C., 2015. In-plane nonlinear localised lateral buckling of straight pipelines. Engineering Structures 103, 37-52. 